\title{
SNARE-Encoding Genes VdSec22 and VdSso1 Mediate Protein Secretion Required for Full Virulence in Verticillium dahliae
}

\author{
Jie Wang, ${ }^{1}$ Li Tian, ${ }^{3}$ Dan-Dan Zhang, ${ }^{1}$ Dylan P. G. Short, ${ }^{2}$ Lei Zhou, ${ }^{1}$ Shuang-Shuang Song, ${ }^{3}$ Yan Liu, ${ }^{3}$ \\ Dan Wang, ${ }^{1}$ Zhi-Qiang Kong, ${ }^{1}$ Wei-Ye Cui, ${ }^{1}$ Xue-Feng Ma, ${ }^{1}$ Steven J. Klosterman, ${ }^{4}$ \\ Krishna V. Subbarao, ${ }^{2,+}$ Jie-Yin Chen, ${ }^{1,+}$ and Xiao-Feng Dai ${ }^{1,+}$ \\ ${ }^{1}$ Laboratory of Cotton Disease, Institute of Food Science and Technology, Chinese Academy of Agricultural Sciences, Beijing, \\ 100193, China; ${ }^{2}$ Department of Plant Pathology, University of California, Davis, c/o U.S. Agricultural Research Station, Salinas, \\ CA, U.S.A.; ${ }^{3}$ College of Life Science, Qufu Normal University, Qufu, 273165, Shandong, China; and ${ }^{4}$ United States Department \\ of Agriculture, Agricultural Research Service, Salinas, CA, U.S.A.
}

Accepted 5 February 2018.

\begin{abstract}
Proteins that mediate cellular and subcellular membrane fusion are key factors in vesicular trafficking in all eukaryotic cells, including the secretion and transport of plant pathogen virulence factors. In this study, we identified vesicle-fusion components that included 22 soluble $N$-ethylmaleimide-sensitive factor attachment protein receptors (SNAREs), four Sec1/Munc18 (SM) family proteins, and 10 Rab GTPases encoded in the genome of the vascular wilt pathogen Verticillium dahliae Vd991. Targeted deletion of two SNARE-encoding genes in V. dahliae, VdSec22 and VdSso1, significantly reduced virulence of both mutants on cotton, relative to the wild-type Vd991 strain. Comparative analyses of the secreted protein content (exoproteome) revealed that many enzymes involved in carbohydrate hydrolysis were regulated by VdSec22 or VdSso1. Consistent with a role of these enzymes in plant cell-wall degradation, pectin, cellulose, and xylan utilization were reduced in the VdSec22 or VdSso1 mutant strains along with a loss of exoproteome cytotoxic activity on cotton leaves. Comparisons with a pathogenicity-related exoproteome revealed that several known virulence factors were not regulated by VdSec22 or VdSso1, but some of the proteins regulated by VdSec22 or VdSso1 displayed different characteristics, including the lack of a typical signal peptide, suggesting that $V$. dahliae employs more than one secretory route to transport proteins to extracellular sites during infection.
\end{abstract}

Jie Wang, Li Tian, and Dan-Dan Zhang contributed equally to this work.

${ }^{\dagger}$ Corresponding authors: Xiao-Feng Dai;

E-mail: daixiaofeng_caas@126.com,

Jie-Yin Chen; E-mail: chenjieyin@caas.cn, and

Krishna V. Subbarao; E-mail: kvsubbarao@ucdavis.edu

Funding: This work was supported by the National Key Research and Development Program of China (2017YFD0200601; 2017YFD0201900), the China Natural Scientific Foundation (No. 31671986, 31501600, 31501588 and 31200113), the CAAS (an Agricultural Science and Technology Innovation Program grant to X.F.D), the Special Public Welfare Industry Research on Agriculture (201503109), the Fundamental Research Funds for Central Non-profit Scientific Institution (Y2016CG11, S2016JC05, S2016CG01)

*The $\boldsymbol{e}$-Xtra logo stands for "electronic extra" and indicates that two supplementary figures and eight supplementary tables are published online.

○ 2018 The American Phytopathological Society
Eukaryotic cells use complex intracellular trafficking (vesicular trafficking) pathways for sorting materials to different cellular sites. Protein localization and movement, signal transduction, and multiple developmental processes occur via distinct exocytic and endocytic pathways (Bonifacino and Glick 2004; Samaj et al. 2006; Yao and Xue 2011). Vesicular trafficking can be divided into four essential steps that include vesicle budding, transport, tethering, and fusion. The processes are conserved between yeast and humans (Bonifacino and Glick 2004; Cai et al. 2007; Wickner 2010) and are tightly regulated to ensure delivery of protein cargo to the appropriate cellular compartment.

Key components involved in vesicle fusion identified to date include SNAREs (soluble $N$-ethylmaleimide-sensitive factor attachment protein receptors), tethering complexes, SM (Sec1/Munc18) family proteins, and Rab GTPases (Koumandou et al. 2007; Ostrowicz et al. 2008). The SNARE proteins function as mediators of fusion between vesicular and target membranes. Therefore, the SNAREs are classified as either vesicle-associated or target membrane-associated ( $\mathrm{v}$ - and t-SNAREs, respectively) proteins (Lipka et al. 2007; Ungar and Hughson 2003). SNAREs are further categorized by the sequence similarity of their SNARE domains and by the presence of conserved glutamine (Q) or arginine (R) amino acid residues in the central portion of the domain, subdividing them into the four classes Qa-, Qb-, Qc-, and R-SNAREs (Fasshauer et al. 1998; Ungar and Hughson 2003). SNARE proteins that mediate vesicle fusion are wellstudied; there are at least 24 known SNARE proteins in Saccharomyces cerevisiae (Burri and Lithgow 2004; Kienle et al. 2009), 62 in Arabidopsis thaliana (Sanderfoot 2007), and 37 in Homo sapiens (Hong 2005).

As in other eukaryotes, fungi depend on complex trafficking pathways that employ SNAREs to facilitate the sorting of proteins to their final intracellular or extracellular locations. Vesicular trafficking in the secretory pathway involves two distinct steps, transport between the ER and the Golgi apparatus and between the Golgi apparatus and the plasma membrane (PM). The yeast v-SNARE protein Sec2p facilitates trafficking between the ER and the Golgi apparatus, while the t-SNARE Sso1 facilitates trafficking between the Golgi apparatus and the PM SNARE complex (Bennett 1995; Pelham 2001) for fusion of secretory vesicles at the PM (Aalto et al. 1993; Zhang and Shin 2006). In this model yeast system, Sec22p is involved in anterograde and retrograde transport between the ER and Golgi 
apparatus (Flanagan et al. 2015; Newman et al. 1990). Secretory routes in eukaryotic cells are sometimes classified as conventional secretion systems that deliver signal peptide-containing secretory proteins from the endoplasmic reticulum (ER) to the Golgi complex and then to secretory granules before their release into the extracellular space (Palade 1975; Schekman 2010). The unconventional, Golgi-independent secretion system, transfers certain signal peptide-containing proteins or signal-less cytoplasmic proteins to the extracellular space (De Marchis et al. 2013; Drakakaki and Dandekar 2013).

Studies on the proteins involved in vesicular transport in plant pathogens have revealed that certain proteins of the transport machinery are required for pathogenicity or full virulence. In some instances, the secretory route is clearly established. Effector proteins of filamentous fungi typically follow the conventional secretory route, marked by production in the ER and secretion through Golgi-derived vesicles (de Jonge et al. 2011). Specific SNAREs are known to mediate the transportation and secretion of extracellular proteins important for pathogenesis, such as MoSso1, MoSec22, and MoVamp7 in Magnaporthe oryzae (Dou et al. 2011; Giraldo et al. 2013; Song et al. 2010), Sso2p and Sec9p in Candida albicans (Bernardo et al. 2014), and FgSso1, FgVam7, and FgVps39 in Fusarium graminearum (Li et al. 2017). Disruption of M. oryzae MoSec22, a S. cerevisiae Sec22 homolog, affected extracellular laccase and significantly reduced virulence on rice plants (Song et al. 2010). In Colletotrichum orbiculare, delivery of effector DN3 is blocked in the absence of Sec22, demonstrating its secretion via exocytosis is coupled with Sec22-mediated traffic (Irieda et al. 2014). The M. oryzae SNARE MoSso1 is required for cytoplasmic effector secretion and pathogenesis and relies on an unconventional secretory pathway (Giraldo et al. 2013). In spite of the important roles SNAREs Sec22 and Sso1 play in the secretory pathway, the role of these proteins in exoproteome production and fungal pathogenesis is still unknown.

Verticillium dahliae is a soilborne fungal plant pathogen that causes a widespread and destructive vascular wilt disease on many hosts, including economically important crops (Klosterman et al. 2009). Recent insights into the pathogenesis of $V$. dahliae on various hosts implicates a significant role for the exoproteome in pathogenesis (Chen et al. 2016; Chu et al. 2015; Zhang et al. 2017a and b). Several $V$. dahliae extracellular proteins involved in pathogenicity or plant-host interaction have been elucidated, including VdSSP1, a protein identified in highly virulent defoliating strains (Liu et al. 2013), small secreted cysteine-containing protein 7 (VdSCP7) (Zhang et al. 2017a), the glycoside hydrolase 12 proteins VdEG1 and VdEG3 (Gui et al. 2017), the avirulence protein Ave1 (de Jonge et al. 2012), the chitin-binding lysine motif (LysM) effectors (Kombrink et al. 2017), and the unconventionally secreted effector VdIsc1 (Liu et al. 2014). Of the 44 genes encoding vesicle-fusion components in $V$. dahliae, 22 are classified in the SNARE superfamily (Yang et al. 2013). Certain key SNARE proteins are known to be important in delivering secreted proteins associated with pathogenicity (Tian et al. 2015; Zhou et al. 2017). For example, the SNARE VdSec22 is involved in secretion of extracellular proteins at penetration interfaces in $V$. dahliae (Tian et al. 2015; Zhou et al. 2017). However, a global picture of the entire exoproteome regulated by specific trafficking components is lacking. Recently, the genome of a highly virulent $V$. dahliae strain, Vd991, from cotton was published (Chen et al. 2018).

The main objectives of the current study were to i) identify vesicle-fusion components in the newly acquired genomic sequence of V. dahliae Vd991 (Chen et al. 2018), ii) determine the role of two V. dahliae SNARE orthologuous genes, Sec22 and Ssol (named VdSec22 and VdSsol, respectively) in pathogenicity, iii) determine the contents of the exoproteome mediated by VdSec22 and VdSso1, and iv) elucidate the network of VdSec22and VdSso1-mediated exoproteome involved in pathogenicity.

\section{RESULTS}

Identification of the vesicle-fusion components in Verticillium dahlia.

The reference genome of $V$. dahliae VdLs.17 (version ABJE00000000.1) (Klosterman et al. 2011) encodes 22 SNAREs, four SM family proteins, and 10 Rab GTPases, based on recognizable structural features of the vesicle components (Yang et al. 2013). To identify vesicle-fusion components in the genome of V. dahliae Vd991, which originated from cotton (Chen et al. 2018), we performed a BLASTp search against the full complement of Vd991-encoded proteins, using the known vesicle-fusion components from the reference VdLs.17 genome as queries. The results showed that the vesicle-fusion components in the Vd991 genome are conserved relative to VdLs.17, similarly encoding 22 SNAREs (six Qa-, four Qb-, six Qc-, one Qbc-, and five R-types), four SM family proteins, and 10 Rab GTPases (Supplementary Table S1). BLASTn analysis revealed high sequence identities of vesiclefusion components between Vd991 and VdLs.17 strains; the lowest identity was VdRab6, which shared $94.39 \%$ identity between Vd991 (VEDA_09703) and VdLs.17 (VDAG_10156). However, the vesicle-fusion components displayed divergence at the peptide level, especially VdSft1, VdBet1, VdRab6, and VdRab7. Moreover, the nucleotide and peptide sequence lengths differed for most of the vesicle-fusion components (Supplementary Table S2). For example, the sequence lengths of VdVam7 homologs in VdLs.17 and Vd991 were significantly different, although the value of identities in nucleotide and peptide levels were up to 98.49 and $98.2 \%$ (Supplementary Fig. S1), respectively. These results suggested that, while vesicle-fusion components are broadly conserved across strains of $V$. dahliae, intraspecific sequence divergence and length polymorphisms exist.

A putative model of the associations of SNARE complexes, Rab proteins, and SM proteins associated with vesicle trafficking in $V$. dahliae was constructed by identifying vesiclefusion component orthologs between $V$. dahliae and those of $S$. cerevisiae (Fig. 1). Only minor differences were observed between the $V$. dahliae model and that of the yeast. $V$. dahliae lacks Vam3p (Wada et al. 1997), which mediates vesicle-fusion trafficking from endosomes to vacuoles (Fig. 1). The number of genes encoding vesicle-fusion components is highly conserved between $V$. dahliae and yeast.

\section{The two vesicle-fusion components VdSec22 and VdSso1 are required for full virulence of Verticillium dahliae strain Vd991 on cotton.}

Vesicle-fusion components play a central role in extracellular trafficking of proteins and, therefore, may be necessary for the transport of effectors and other proteins important for pathogenesis in V. dahliae. Certain vesicle-fusion components (Qa, VdSed5; Qb, VdBos1; Qc, VdBet1; R, VdSec22/VdYkt6; SM, VdSly1; Rab, VdRab1) are predicted to participate in trafficking from the ER to the cis-Golgi, while others mediate the movement of secretory vesicles to the PM (Qa, VdSso1/2; Qb/Qc, VdSec9; R, VdSnc1; SM, VdSec1; Rab, VdSec4) (Fig. 1). To assess the role of vesicle-fusion components in $V$. dahliae pathogenesis, mutants of two vesicle-fusion components involved in trafficking from the ER to the cis-Golgi (R, VdSec22) and secretory vesiclefusion to the PM (Qa, VdSso1) were generated in the wild-type strain Vd991 by gene replacement with a hygromycin resistance cassette, via homologous recombination. The susceptible cotton variety was inoculated with three independent $V d S e c 22$ and VdSSol deletion mutants ( $\triangle \operatorname{Sec} 22-1, \Delta \operatorname{Sec} 22-2, \Delta \operatorname{Sec} 22-3$ and $\Delta$ Ssol-1, $\Delta$ Ssol-2, $\Delta$ Ssol-3) along with the wild-type strain Vd991, using a root-dip method. As expected, the VdSec22 deletion strains were less virulent on cotton, whereas the wild-type strain Vd991 caused severe wilting symptoms, including wilting, 
chlorosis, stunting, and vascular discoloration (Fig. 2A). A quantitative polymerase chain reaction (qPCR) analysis of fungal biomass in planta of $\mathrm{VdSec} 22$ deletion strains revealed only about $40 \%$ of the fungal biomass relative to wild-type strain Vd991 (Fig. 2B). Similarly, targeted replacement of VdSsol also significantly reduced virulence and fungal biomass on cotton (Fig. 2C and D). Furthermore, two independent complementation transformant strains revealed that reintroduction of $V d S e c 22$ and $V d S s o l$ with native promoter to $\Delta S e c 22-1$ and $\Delta S$ sol-1 mutants, respectively, restored their virulence (vascular discoloration and fungal biomass), comparable to that of the wild-type strain when inoculated on cotton (Supplementary Fig. S2). These results therefore suggested that $\mathrm{VdSec} 22$ or $\mathrm{VdSso1}$ is required for full virulence of V. dahliae Vd991 on susceptible cotton (cv. Junmian 1), indicating that the vesicle-fusion components involved in trafficking play important roles in the pathogenicity and virulence of $V$. dahliae.

\section{Deletion of VdSec22 or VdSso1 reduces catabolism of multiple carbon sources.}

Based on homology to known orthologs described above, the concerted functions of $\mathrm{VdSec} 22$ or $\mathrm{VdSso} 1$ were hypothesized to mediate vesicle trafficking and translocation of proteins from intracellular to extracellular space. These candidate proteins in $V$. dahliae include multiple pectinases, cellulases, and xylanases, predicted with extracellular localization (Klosterman et al. 2011). To identify the functional exoproteome regulated by $\mathrm{VdSec} 22$ or
VdSso1, the ability of the strains $\Delta S e c 22$ and $\Delta S s o 1$ to digest carbon sources was monitored using three major plant cell-wall components, including pectin, cellulose, and xylan. Wild-type strain Vd991 grew well on all carbon sources tested (Fig. 3A). However, the growth of the $\Delta \operatorname{Sec} 22$ and $\Delta S$ sol mutants was significantly restricted on media that contained the different plant cell-wall components as carbon sources (Fig. 3A). Wild-type strain Vd991 in media containing all cell-wall components covered the entire plate (35 to $45 \mathrm{~mm}$ ) in 10 days, but the $V d S e c 22$ and VdSsol deletion mutants only formed a small colony (15 to $20 \mathrm{~mm}$ ) during this period (Fig. 3B). Unexpectedly, strain $\Delta$ Sec22 displayed a darker phenotype characteristic of melanin biosynthesis on cellulose, whereas wild-type Vd991 did not produce melanin on cellulose (Fig. 3A). Together, these results indicated that the exoproteome regulated by $\mathrm{VdSec} 22$ or VdSso1 contained enzymes involved in catabolism of multiple carbon sources, including several plant cell-wall components, such as pectin, cellulose, and xylan.

\section{The exoproteome regulated by VdSec22 and VdSso1 affects cytotoxic activity on cotton leaves.}

Previous studies in our laboratory (Chen et al. 2016) showed that the $V$. dahliae exoproteome induced by cotton-containing medium compared with the basic medium (Czapek-Dox medium) was associated with wilting activity on cotton leaves. To evaluate the cytotoxic activity of the secreted proteins regulated by

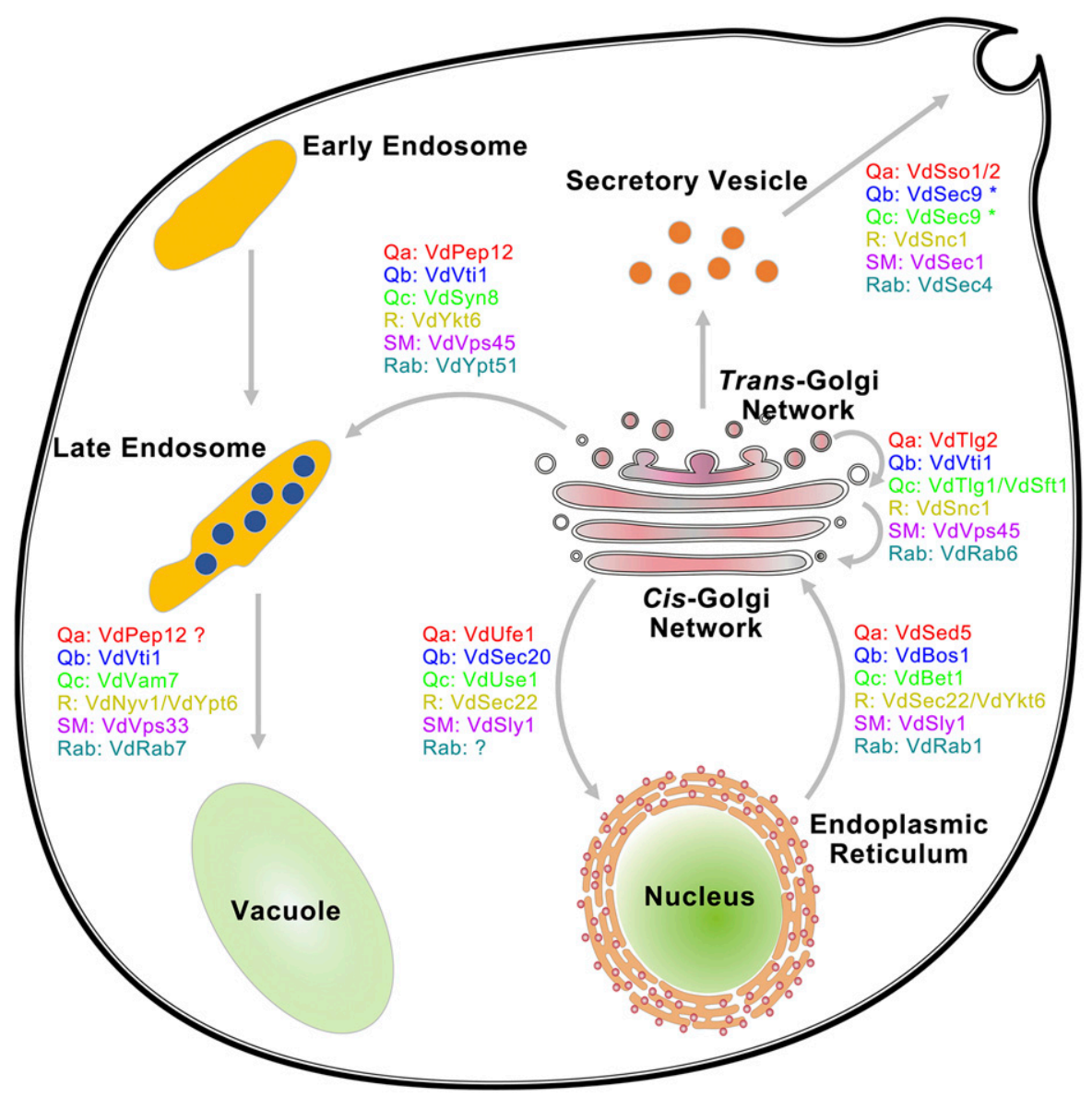

Fig. 1. Prediction of the association of $N$-ethylmaleimide-sensitive factor attachment protein receptor (SNARE) complexes, Rab proteins, and Sec1/Munc18 (SM) proteins with trafficking steps in Verticillium dahliae. The SNAREs, SM family proteins, and Rab GTPases encoded in the V. dahliae Vd991 genome were determined by the ortholog similarity analysis with the SNARE proteins, Rab proteins, and SM proteins in the VdLs.17 genome (Yang et al. 2013). The association of SNARE complexes, Rab proteins, and SM proteins with trafficking steps in V. dahliae were drawn according to the typical trafficking steps in yeast (Jahn et al. 2003; Kienle et al. 2009). Asterisks denote that protein VdSec9 belongs to Qb and Qc types. Qa, Qb, Qc, and R are the four classes of SNAREs. The Qa, Qb, and Qc families contain a conserved glutamine $(\mathrm{Q})$ amino acid residue in the central position of the domain, and the R family contains an arginine $(\mathrm{R})$ residue in the central layer of the domain. 
VdSec22 and VdSso1, the exoproteomes of VdSec22 and VdSsol deletion mutants were purified from a culture suspension and the wilting-activity was evaluated on cotton cotyledons. The induced exoproteome of strain Vd991 purified from cotton-containing medium caused chlorosis and necrosis of cotton leaves 3 days after inoculation (Fig. 4). By 5 days postinoculation, chlorosis and necrosis on cotton leaves became more obvious and the whole cotton leaf was nearly necrotic (Fig. 4). However, compared with the wild-type strain, the exoproteome of $\Delta S e c 22$ and $\Delta S$ sol strains nearly lost cytotoxic activity on cotton leaves, showing almost no wilt-like symptoms 3 days postinoculation (Fig. 4). By 5 days postinoculation, the mutants showed wilt-like symptoms with incipient chlorosis and necrosis on cotton leaves (Fig. 4). These results suggested that the exoproteomes from VdSec22 and VdSso1 regulate the cytotoxic activity observed on cotton leaves.

\section{Identification of the exoproteome regulated by VdSec22 and VdSso1.}

To elucidate which pathogenesis-related components of the exoproteome are regulated by $\mathrm{VdSec} 22$ and $\mathrm{VdSso1}$, the exoproteomes of $V d S e c 22$ or $V d S s o l$ deletion strains were compared with the wild-type strains grown in cotton-containing medium, as determined by isobaric tags for relative and absolute quantitation (iTRAQ) methods. A total of 461 and 383 putative proteins were identified using a concatenated targetdecoy strategy with Vd991 genome sequences and analyses of the VdSec 22 or VdSsol deletion strains (Supplementary Table S3). The abundance of 255 (120 proteins downregulated) and 162 (61 proteins downregulated) proteins were significantly altered (abundance fold change $\geq 1.5, P<0.01$ ) in $V d S e c 22$ and $V d S s o 1$ deletion strains, respectively, compared with the wildtype strain (Fig. 5A; Supplementary Table S4). Of the regulated exoproteome, 79 proteins were common between $V d S e c 22$ and $V d S s o 1$ deletion mutants, of which 43 and 25 proteins were down- or upregulated in $V d S e c 22$ and VdSsol deletion mutants (Fig. 5A), respectively. The fold change ranges of the VdSec22and VdSsol-regulated exoproteomes revealed that the abundance of downregulated proteins (change of half of them greater than 2.27- and 2.03-fold, respectively) was slightly higher than that of the upregulated protein set (change of half of

A Mock

$\Delta \operatorname{Sec} 22-1$

$\Delta \operatorname{Sec} 22-2$

$\Delta \operatorname{Sec} 22-3$

WT
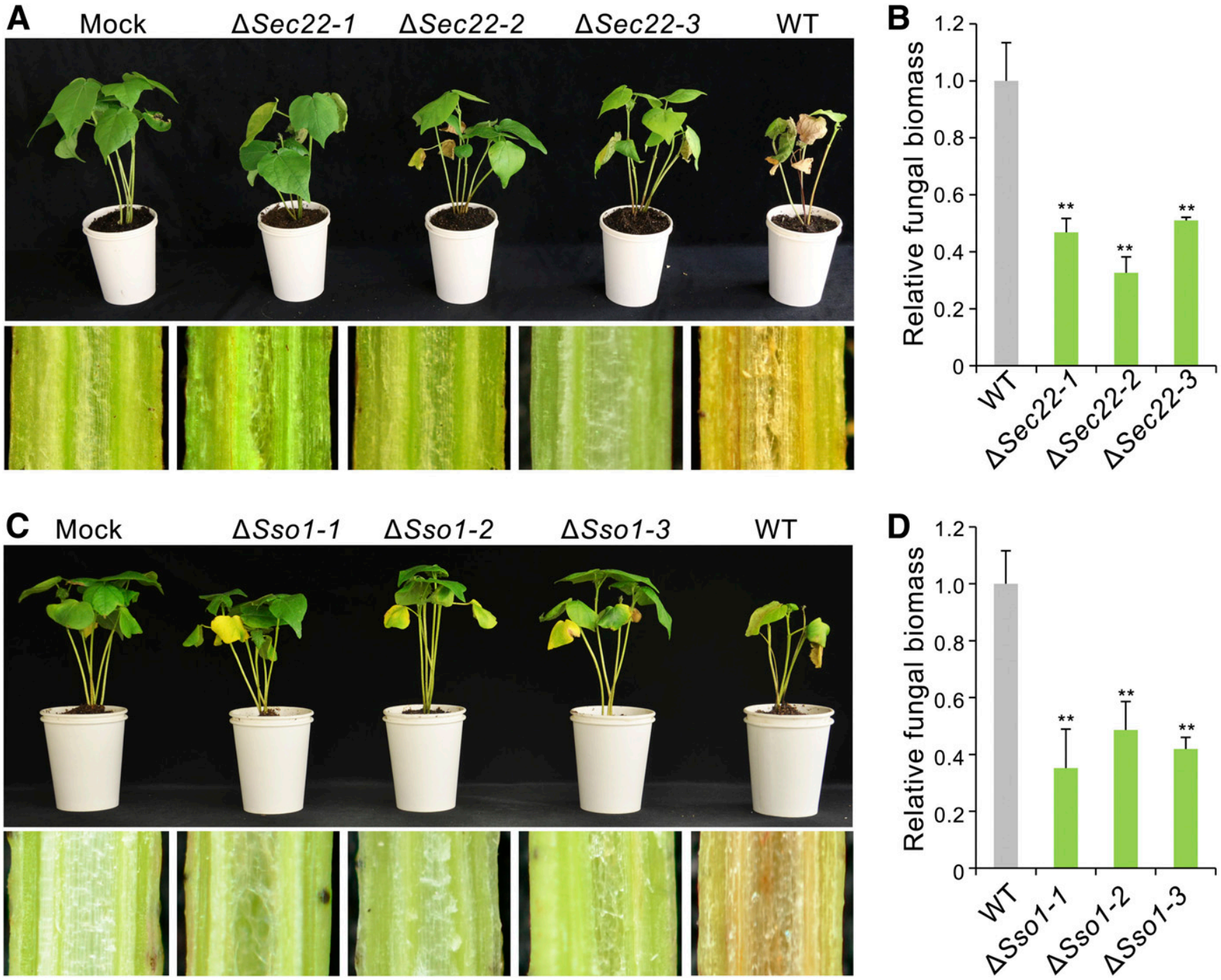

Fig. 2. The vesicle-fusion components of VdSec22 and VdSso1 are required for the pathogenicity of Verticillium dahliae on cotton. A, Phenotypes of cotton seedlings inoculated with VdSec22 and C, VdSsol gene-deletion strains. Two-week-old seedlings of susceptible cotton (cv. Junmian 1) were inoculated with sterile water (Mock), wild-type $V$. dahliae (WT), VdSec22 gene-deletion strains ( $\Delta \operatorname{Sec} 22-1, \Delta \operatorname{Sec} 22-2$, and $\Delta S e c 22-3)$ in A and $V d S s o 1$ gene-deletion strains ( $\Delta$ Sso1-1, $\Delta$ Sso1-2, and $\Delta S s o 1-3$ ) in C. Verticillium wilt symptoms (top) were photographed 3 weeks after plant inoculation, and the discoloration of the inoculation shoot longitudinal sections is shown at the bottom. B, The fungal biomasses of $V d S e c 22$ and $\mathbf{D}$, VdSsol gene deletion strains on cotton were determined by quantitative polymerase chain reaction. Error bars represent standard errors. Asterisks $(* *)$ indicate significant differences $(P<0.01)$, according to unpaired Student's $t$ test. 
them greater than 2.00- and 1.92-fold, respectively) (Fig. 5B), suggesting that the regulation of exoproteome by $\mathrm{VdSec} 22$ and VdSsol occurred mainly by upregulation of their respective targets.

Gene ontology (GO) analysis revealed that the exoproteomes were enriched in catalytic activity under GO ID 0003824, at a ratio of $58.0 \%(P<0.001), 45.7 \%(P<0.001)$, and $64.6 \%(P<$ $0.05)$ in VdSec22- and VdSso1-regulated exoproteomes and the common proteins regulated by $\mathrm{VdSec} 22$ and $\mathrm{VdSso1}$, respectively (Fig. 5C). Most of the proteins associated with this catalytic activity (GO:0003824) have hydrolase activity (GO: 0016787), and constitute a high proportion of proteins- $65.5 \%$ (97 proteins, $P<0.001$ ), 63.5\% (47 proteins, $P<0.001$ ), and $62.7 \%$ (32 proteins, $P<0.001$ ) in the VdSec22- and VdSso1regulated exoproteomes and the common proteins regulated by VdSec22 and VdSsol (Fig. 5C), respectively. The proteins identified with hydrolase activity (GO:0016787) participate in the carbohydrate metabolism (GO:0016798, acting on glycosyl bonds, $P<0.01$ ) (Fig. 5C). Together, these results suggested that the exoproteomes regulated by $\mathrm{VdSec} 22$ and VdSso1 exhibit numbers of proteins with disproportionately higher hydrolase function.

\section{Functional analysis of the exoproteome regulated by VdSec22 and VdSso1.}

Characteristics of the VdSec22 and VdSso1 exoproteomes were further analyzed by additional bioinformatics-driven methods, including the detection of typical secretion signals (signal peptide analysis), detection of carbohydrate-active enzymes (CAZymes), detection of small cysteine-rich proteins (SCRPs, $<400$ amino acids, $\geq 4$ cysteine residues), and the detection of homologs of known pathogen-host interaction (PHI) proteins. Of the VdSec22- and VdSso1-regulated exoproteomes and their commonly regulated proteins, more than half (53.1, 58.9 , and $72.5 \%$, respectively) the total regulated proteins contain a signal peptide (Table 1; Supplementary Table S5). Functional annotation showed that $\mathrm{VdSec} 22$ and $\mathrm{VdSso} 1$ and their commonly regulated proteins mainly were involved in
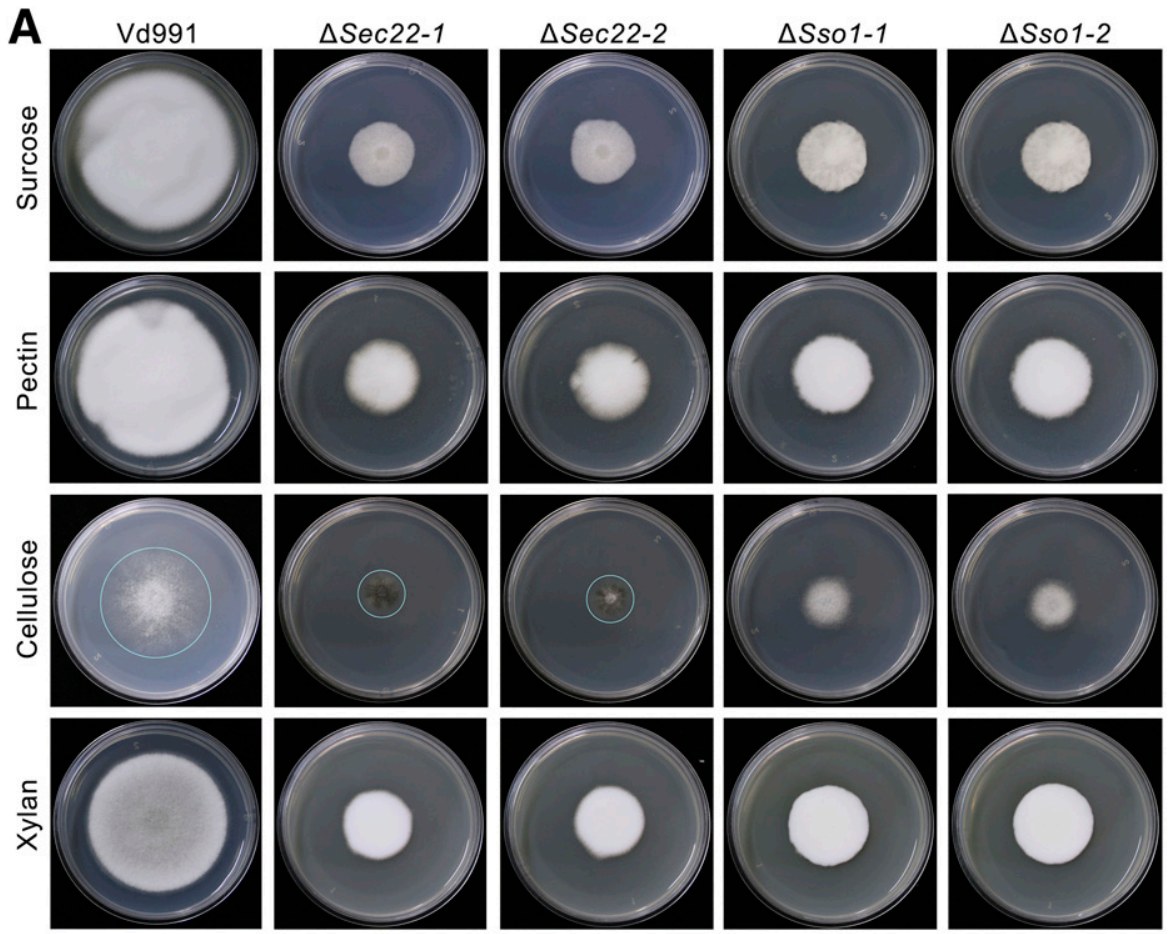

B

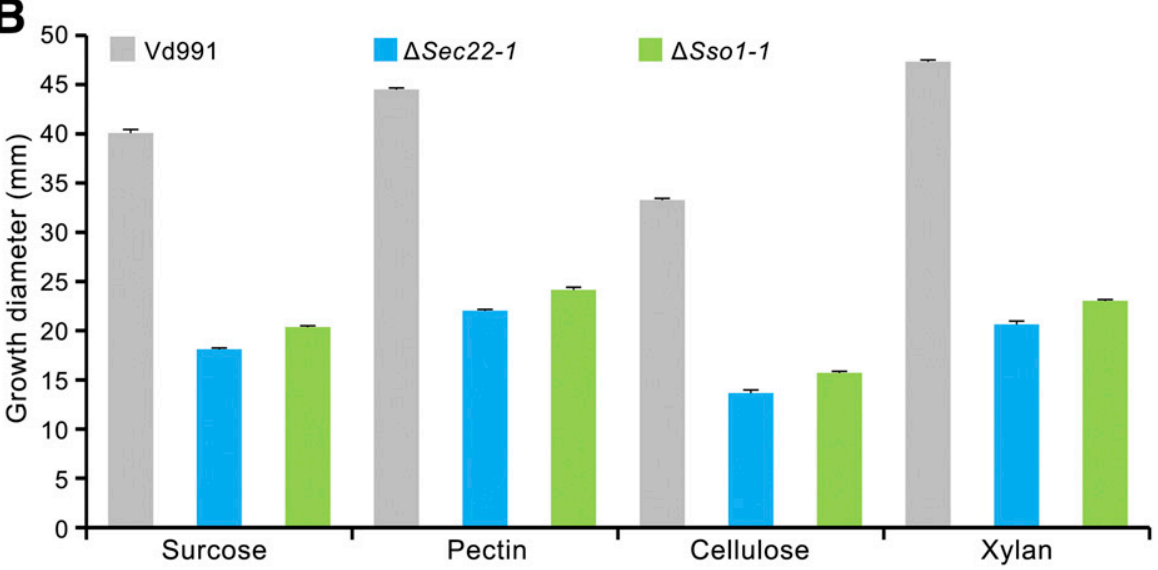

Fig. 3. Analyses of carbon source utilization in the deletion mutants VdSec22 or VdSsol of Verticillium dahliae. A, The VdSec22 or VdSso1 deletion mutant strains grew on the plant cell-wall components of pectin, cellulose, and xylan. Ten microliters of a $5 \times 10^{6}-$ conidia/ml suspension was dropped on the center of the plate and was incubated at $25^{\circ} \mathrm{C}$ for 10 days. B, Growth diameter of the VdSec 22 or $V d S s o l$ mutants was determined on the media containing different carbon sources at 10 days after incubation. 
carbohydrate metabolism, which were predicted as CAZymes, and many of these were typically secreted proteins in ratios of 42.2 , 39.9 , and $60.0 \%$ (Table 1), respectively. In addition, several proteins regulated by $V d S e c 22$ and $V d S s o l$ and their shared regulated proteins were also predicted as SCRPs and PHI homolog proteins (Table 1), associated with pathogenicity.

Most of the CAZymes were down-regulated following deletion of either $V d S e c 22$ or $V d S s o 1$, with 87 and 65 proteins (43 in common) downregulated, respectively (Table 1). VdSec22 and VdSsol regulated all the major CAZyme classes, including those of the following families: five auxiliary activity, two carbohydrate-binding module, 10 carbohydrate esterases, 33 glycoside hydrolases (GHs), and four polysaccharide lyases (PLs) (Table 2; Supplementary Table S6). In the CAZymes class of the GHs, this was especially apparent and included 61 and 37 proteins (25 proteins in common) regulated by, respectively, VdSec22 and VdSso1 (Table 2). Among the class of PLs, 13 and 12 proteins (nine proteins in common) were regulated by VdSec22 and VdSso1 (Table 2), respectively. Plant cellwall degradation may occur through the action of CAZyme families regulated by $\mathrm{VdSec} 22$ and $\mathrm{VdSso1}$, including those that mediate pectin and cellulose degradation. In total, 29 and 24 proteins (18 proteins in common) of the identified proteins regulated by $\mathrm{VdSec} 22$ and $\mathrm{VdSso1}$, respectively, were associated with pectin degradation and 22 and nine proteins (eight proteins in common) were associated with cellulose degradation (Table 2), respectively. Together, these results suggested that $\mathrm{VdSec} 22$ and $\mathrm{VdSso1}$ regulate groups of typically secreted proteins involved in carbohydrate (pectin and cellulose) degradation.

VdSec22 and VdSso1 regulate the exoproteome involved in plant cell-wall degradation and pathogenesis.

Previous work revealed that the Vd991 pathogenesis-related exoproteome was composed of 271 proteins (161 are typical secreted proteins) and that 126 of them belonged to CAZyme (106 are typical) families (Chen et al. 2016). The secreted CAZymes or their functional associations are typically upregulated (Fig. 6A). Comparison of the VdSec22 and VdSso1 exoproteomes to the previously published pathogenicity-related exoproteome revealed that a large portion of the pathogenicityrelated exoproteome was also regulated by $\mathrm{VdSec} 22$ and VdSso1, including 115 (89 are typical) and 72 (57 are typical) proteins (Fig. 6A), respectively. VdSec22 and VdSso1 regulated 78 (69 are typical) and 44 (41 are typical) proteins that were CAZymes of the pathogenicity-related exoproteome (Fig. $6 \mathrm{~A})$, respectively. Of the pathogenicity-related exoproteomes commonly regulated by $\mathrm{VdSec} 22$ and VdSso1, these included 43 proteins (36 are typical), in which 31 are CAZymes (29 are typical) (Fig. 6A). The pathogenicity-related exoproteome regulated by $\mathrm{VdSec} 22$ and $\mathrm{VdSso} 1$ was especially apparent in the pathogenicity-related exoproteome regulated by $V d S e c 22$, which included 75 proteins (69 are typical) that were significantly down-regulated in VdSec22 deletion mutants. Most of these were determined to be CAZymes (Fig. 6A). Further investigation of the CAZymes in the pathogenicity-related exoproteomes regulated by $\mathrm{VdSec} 22$ and $\mathrm{VdSso} 1$ revealed that many were associated with plant cell-wall degradation (pectin, cellulose, xylan, xylogulan, and more) and were primarily up-regulated in these exoproteomes (Fig. 6B). CAZyme protein abundance was significantly up-regulated by cottoncontaining medium but was significantly down-regulated after deletion of either $V d S e c 22$ or $V d S s o 1$ (Fig. 6C). For example, 31 proteins associated with pectin degradation were significantly up-regulated in cotton-containing medium and 20 of them were down-regulated after deletion of $\mathrm{VdSec} 22$ (Fig. $6 \mathrm{~B}$ and $\mathrm{C})$.

\section{Verticillium dahliae employs diverse secretory routes} to translocate the virulence-related exoproteome.

Comparison of the pathogenesis-related exoproteome to the VdSec22 and VdSso1 exproteomes showed that many extracellular proteins (Fig. 6A) were also secreted independent of VdSec22 and VdSso1. Of the pathogenesis-related exoproteome, 127 proteins (51 proteins are the typical secreted proteins) were determined as secreted independent of $\mathrm{VdSec} 22$ and VdSso1, of which 35 are CAZymes (27 proteins are the typical secreted proteins) (Fig. 6A). Investigation of the
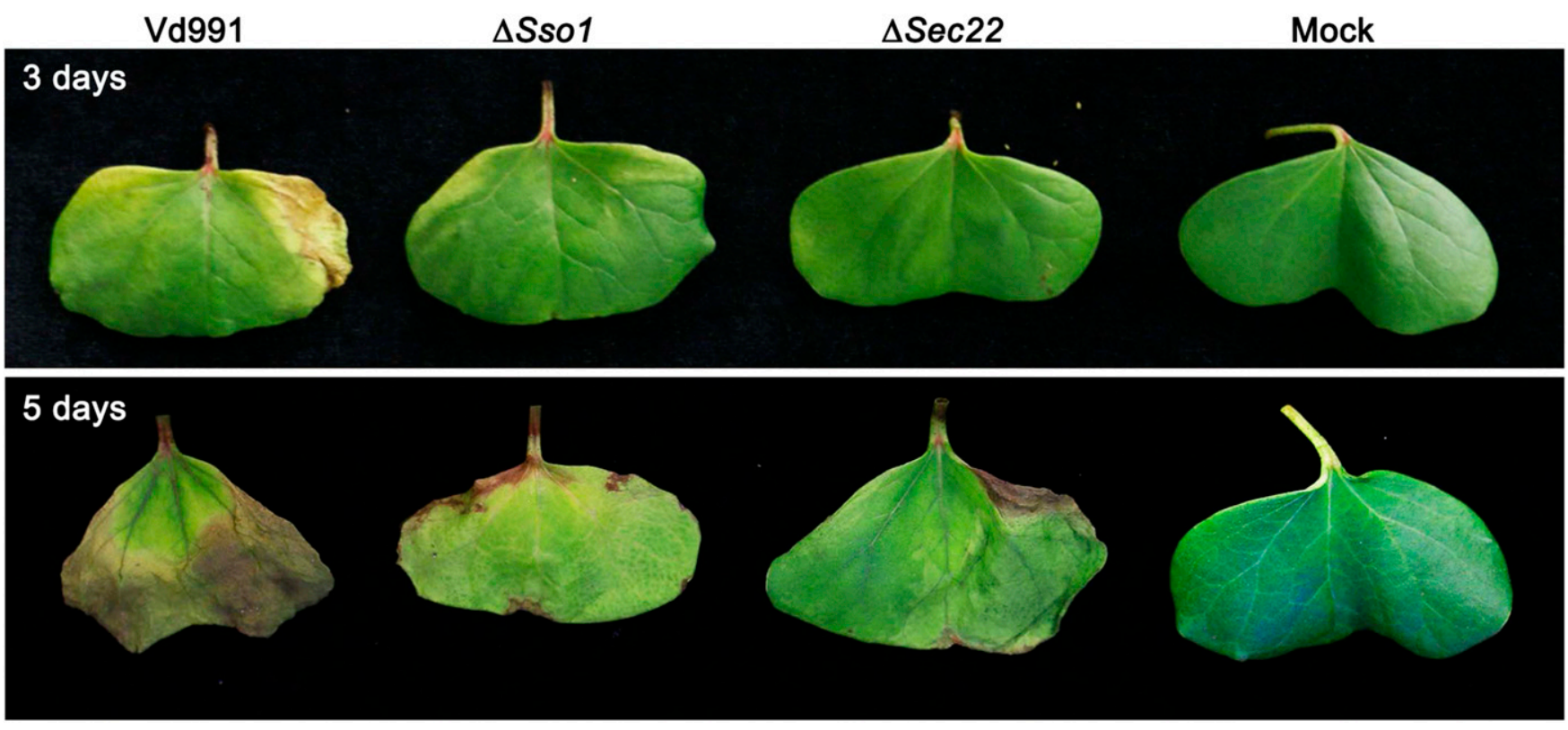

Fig. 4. The exoproteome of $V d S e c 22$ and $V d S s o l$ deletion mutant strains reduced wilting activity on cotton cotyledons. The wilting activity of the exoproetome $(1.0 \mu \mathrm{g})$ derived from the VdSec22 or VdSsol deletion strains and the wild-type strain of Vd991 was assayed on Gossypium hirsutum L. 'Junmian No. 1' cotyledons containing petioles collected from 2 -week-old seedlings. The cotton-containing medium was set as control (Mock). The cotyledon phenotypes were investigated 3 days and 5 days after incubation at $25^{\circ} \mathrm{C}$. 
CAZAymes not regulated by $\mathrm{VdSec} 22$ and $\mathrm{VdSso} 1$ revealed that the protein abundance was significantly up-regulated after culture in the cotton-containing medium, such as a GH3 family protein (VEDA_03908), GH28 (VEDA_01417), CBM20 (VEDA_05547), GH37 (VEDA_09336), and a GH79 family member (VEDA_07224). These particular proteins were up- regulated by a >8-fold change in the wild-type strain Vd991 (Fig. 7A). A previous study of gene expression showed that two of these genes, PL1 (VEDA_01449) and GH3 (VEDA_09734), were significantly up-regulated during infection of cotton (Chen et al. 2016), and targeted gene deletion VEDA_05769, encoding the GH12 family member VdEG3, a known pathogen-associated
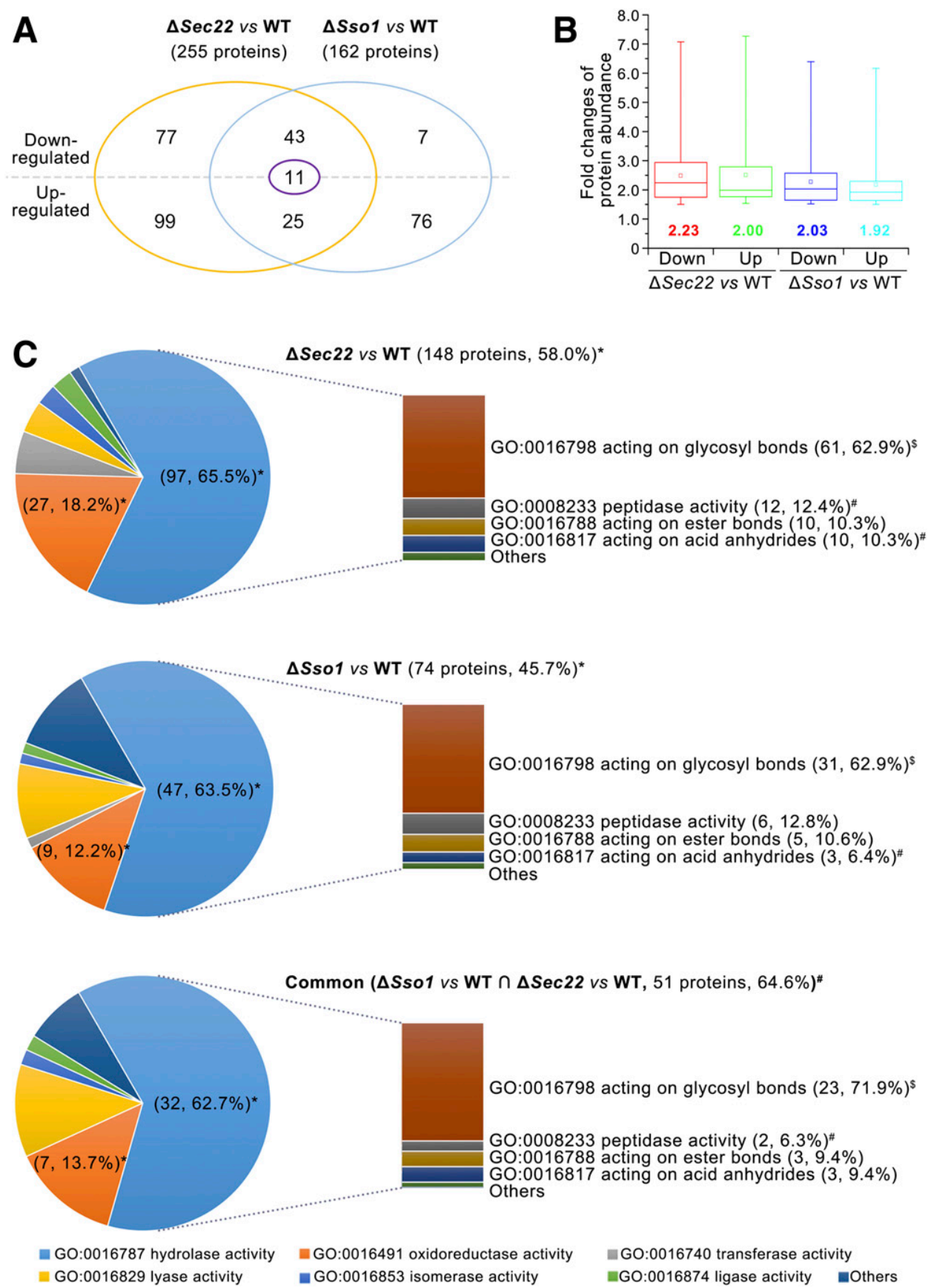

Fig. 5. Identification and functional analysis of Verticillium dahliae exoproteomes regulated by VdSec22 and VdSso1 in cotton tissue-containing medium. A, Statistical analysis of the exoproteome regulated by VdSec22 and VdSso1 in the cotton-containing medium. The orange and blue ellipse represents the exoproteome regulated by VdSec22 and VdSso1, respectively. The numbers up to the dotted line represent the significantly downregulated $(P<0.01,1.5$-fold change) proteins in the cotton-containing medium after deletion of $V d S e c 22$ or $V d S s o l$ in $V$. dahliae, and the numbers down to the dotted line represent significantly upregulated proteins in the cotton-containing medium. The numbers in the purple ellipse represent the proteins that display differential patterns of regulation after deletion of $V d S e c 22$ or VdSsol in V. dahliae, showing those upregulated in the VdSec22 deletion mutant but downregulated in the VdSsol deletion mutant. B, Statistical analysis of the fold change of the identified proteins after deletion of VdSec22 or VdSsol in V. dahliae. The fold-change variation of the identified proteins was displayed by the Box whisker plot. In each box plot, the central line represents the fold-change value of the median number of identified proteins (the value was marked under each box), the rectangle gives the interval between the 25 and $75 \%$ percentiles of the identified proteins, and the whisker indicates the extreme value of the fold change of the identified proteins. C, Gene ontology (GO) functional analysis of the catalytic class (GO: 0003824) of the exoproteome regulated by VdSec22 and VdSso1 in cotton tissue-containing medium. The significant differences of GO enrichment were analyzed by hypergeometric test for each GO term and were subsequently corrected for multiple testing error, with symbols *, \$, and \# indicating significant differences of $P$ value $<0.001,<0.01$, and $<0.05$, respectively. 
molecular pattern, reduced virulence on cotton (Gui et al. 2017). In addition, of the 76 proteins without secretory characteristics and not regulated by $\mathrm{VdSec} 22$ and $\mathrm{VdSso1}$, several were also significantly up-regulated. Some of these had known roles in pathogenesis, along with CAZymes and PHI homologs (Supplementary Table S7). Verticillium dahlia, thus, employs different secretory routes, at least one of which may be independent of $\mathrm{VdSec} 22$ and VdSso1, to translocate the pathogenesis-related exoproteome (Fig. 7B), including relevant CAZymes. Nevertheless, VdSec22 and VdSso1 regulate the secretory routes for exoproteome secretion, including those proteins also important for pathogenicity (Fig. 7B).

\section{DISCUSSION}

The vascular wilt fungi and other pathogens secrete an arsenal of effector proteins during plant colonization that are capable of targeting multiple host cell compartments (de Sain and Rep 2015; Franceschetti et al. 2017). Vesicle-fusion components, which mediate protein transport to the extracellular space, can play a critical role in pathogenicity (Bernardo et al. 2014; Dou et al. 2011; Giraldo et al. 2013; Song et al. 2010). In this study, we provided evidence for the critically important role of two SNARE genes, VdSec22 and VdSso1, in protein secretion and virulence in the $V$. dahliae Vd991. This study demonstrates that the exoproteome regulated by the vesiclefusion components VdSec22 and VdSso1 functions as a virulence factor during infection of cotton, especially the secreted proteins associated with carbohydrate enzymes involved in plant cell-wall degradation. CAZymes regulated by VdSec22 or VdSso1 have previously been implicated in pathogenicity. Targeted deletion of the polysaccharide lyase VEDA_02612 (VdPL3.1) in strain Vd991 significantly reduced virulence on cotton; and several other genes, including PL1 (VEDA_04771 and VEDA_02526), AA9 (VEDA_00597 and VEDA_06755), and $G H 7$ (VEDA_01162 and VEDA_08911) were significantly up-regulated during infection on cotton (Zhang et al. 2017b).

Studies in different eukaryotic organisms have revealed that the principal organization of the endomembrane systems and components of the molecular machinery involved in vesicular trafficking are conserved among all eukaryotes, especially the core components of SNAREs contributing to vesicle fusion (Cai et al. 2007; Jahn et al. 2003; Kienle et al. 2009; Pelham 2001). In V. dahliae, the vesicle-fusion components have been well-studied in the reference genome VdLs.17, and this genome encodes conserved vesicle-fusion components relative to the same machinery in yeast and also similar to other fungal species (Yang et al. 2013), such as the presence of the fungalspecific SNARE protein Vam7 (Kienle et al. 2009). This was also the case in the genome of $V$. dahliae Vd991 from cotton. The sequences among VdLs.17 and Vd991 strains displayed comparable divergence, even though both contained (Qc type SNAREs VdSft1 and VdBet1, which may affect the efficiency of vesicle fusion in different $V$. dahliae strains. Similarly, the homolog of yeast Vam3p was not found in the Vd991 genome but was reported as encoded in the genome of VdLs.17 (Yang et al. 2013), suggesting a lack of Qa type SNAREs for vesicle fusion from late endosome to vacuole in Vd991. Endosomal syntaxins Pep12p and Vam3p are paralogs that arose from gene duplication (Kienle et al. 2009), and thus, VdPep12 probably overlaps the functional role of the Qa-type SNAREs during vesicle fusion of late endosomes to vacuoles in $\mathrm{Vd} 991$.

In fungi, some vesicle-fusion components, like RabGTPases, appear to be highly divergent or species-specific, indicating that fungal vesicle fusions might have specialized or general functions (Yang et al. 2013). Regarding VdVam7, the sequence length of the protein from Vd991 strain is similar to that of the SNAREs from other fungi, confirming that the sequence is truncated in the published VdLs.17 genome (version ABJE00000000.1) (Klosterman et al. 2011). The vesicle-fusion components are conserved within isolates of $V$. dahliae, but the sequence and genetic divergence is observed in SNAREs, which probably affects the efficiency of vesicle fusion during cellular processes.

Previous studies have shown that various homologs of vesicle components are critical in the infection of host plants by pathogenic fungi, and several of them were shown to govern virulence and pathogenicity, including the SNARE components of MoVam7 and MoSec22 from M. oryzae (Dou et al. 2011; Song et al. 2010), the SNAREs FgVamp7 and FgSso1 from Fusarium graminearum (Li et al. 2017; H. Zhang et al. 2016), the conserved SNARE PsYKT6 from Phytophthora sojae (Zhao et al. 2011), and the syntaxin MoSyn8 from M. oryzae (Qi et al. 2016). In V. dahliae, analysis of the expression profiles of the genes encoding vesicle components indicated that many of them are significantly differentially expressed during vegetative growth and microsclerotia formation (Yang et al. 2013). In this study, the two components of SNAREs, VdSec22 and VdSso1, significantly contributed to pathogenicity on cotton, while $\mathrm{VdSec} 22$ had previously been shown to play a critical role in $V$. dahliae pathogenicity (Tian et al. 2015; Zhou et al. 2017). Similarly, in M. oryzae, the SNARE components of MoSec22 also contributed to pathogenicity during infection of rice plant (Song et al. 2010). Although the reduction in the growth rate of $V d S e c 22$ and VdSsol mutant strains may, in part, contribute to defects in plant infection, other important pathogenicity factors, including those involved in plant cell-wall degradation, are also affected in the mutant that may result in reduced virulence. Additional

Table 1. Statistical analysis of the exoproteome of Verticillium dahliae regulated by VdSec22 and VdSso1 in cotton tissue-containing medium ${ }^{\text {a }}$

\begin{tabular}{|c|c|c|c|c|c|c|c|c|}
\hline \multirow{2}{*}{$\frac{\text { Functional }}{\text { Regulation types }}$} & \multicolumn{2}{|c|}{ Secreted proteins } & \multicolumn{2}{|c|}{ CAZymes $^{b}$} & \multicolumn{2}{|c|}{$\operatorname{SCRPs}^{\mathrm{c}}$} & \multicolumn{2}{|c|}{ PHI proteins ${ }^{d}$} \\
\hline & Down & Up & Down & Up & Down & Up & Down & Up \\
\hline$\Delta \operatorname{Sec} 22$ vs. WT & \multicolumn{2}{|c|}{136 proteins, $53.1 \%$} & \multicolumn{2}{|c|}{108 (91] proteins, $42.2 \%$} & \multicolumn{2}{|c|}{11 proteins, $5.3 \%$} & \multicolumn{2}{|c|}{28 (19) proteins, $10.9 \%$} \\
\hline 256 proteins & 107 & 29 & $87(79)$ & $21(12)$ & 8 & 3 & $20(18)$ & $8(1)$ \\
\hline$\Delta$ Ssol vs. WT & \multicolumn{2}{|c|}{96 proteins, $58.9 \%$} & \multicolumn{2}{|c|}{$65(58]$ proteins, $39.9 \%$} & \multicolumn{2}{|c|}{11 proteins, $6.7 \%$} & \multicolumn{2}{|c|}{16 (13) proteins, $9.8 \%$} \\
\hline 163 proteins & 44 & 52 & $39(35)$ & $26(23)$ & 4 & 8 & $10(9)$ & $6(4)$ \\
\hline$\Delta \operatorname{Sec} 22$ vs. WT & \multicolumn{2}{|c|}{58 proteins, $72.5 \%$} & \multicolumn{2}{|c|}{$48(43]$ proteins, $60.0 \%$} & \multicolumn{2}{|c|}{11 proteins, $13.8 \%$} & \multicolumn{2}{|c|}{10 (9) proteins, $12.5 \%$} \\
\hline$\Delta S s o l$ vs. WT & 39 & 11 & $37(33)$ & $6(5)$ & 2 & 1 & $8(8)$ & $2(1)$ \\
\hline
\end{tabular}

${ }^{a}$ Functional annotation of the exoproteome regulated by VdSec22 and VdSso1. The typical secreted proteins were defined by proteins with extracellular localization (WoLF PSORT software) that contained a signal peptide and a signal peptide cleavage site (SignalP 4.1 software) but lacking transmembrane domains (TMHMM 2.0 and Phobius software). WT = wild type.

b The carbohydrate-active enzymes (CAZymes) were predicted using the BLAST and hidden Markov model-based routines of the CAZy database.

c The small cysteine-rich proteins (SCRPs) were determined by the typical secreted proteins ( $<400$ amino acids, $\geq 4$ cysteine residues) without known function (conserved/hypothetical proteins).

$\mathrm{d}$ The pathogen-host interaction (PHI) proteins were predicted using the BLAST analysis to the PHI database. The number in parenthesis represents the typical secreted proteins. 
Table 2. Carbohydrate-active enzyme (CAZyme) families classification of the exoproteome of Verticillium dahliae regulated by VdSec22 and VdSso1 in cotton tissue-containing medium ${ }^{\mathrm{a}}$

\begin{tabular}{|c|c|c|c|c|c|c|c|}
\hline Superfamily & Family & Functional & Subfamily & Possible Substract & VdSec22 & VdSso1 & Common \\
\hline \multirow[t]{6}{*}{ AAs } & AA2 & Lignin-modifying peroxidase & AA2 & & 3 & 2 & 2 \\
\hline & AA3 & Glucose-methanol-choline oxidoreductas & AA3 & & 1 & 0 & 0 \\
\hline & AA7 & Glucooligosaccharide oxidases & AA7 & & 3 & 2 & 2 \\
\hline & AA8 & Cytochrome domain & AA8 & & 1 & 0 & 0 \\
\hline & AA9 & Monooxygenase & AA9 & & 4 & 3 & 3 \\
\hline & & & AA9, CBM1 & Cellulose & $2(1)$ & 0 & 0 \\
\hline \multirow[t]{2}{*}{ CBMs } & CBM1 & Cellulose-binding & CBM1 & Cellulose & 1 & 1 & 1 \\
\hline & CBM50 & Targeting the petidoglycan & CBM50 & Chitin; peptidoglycan & 1 & 0 & 0 \\
\hline \multirow[t]{11}{*}{ CEs } & CE8 & Pectin methylesterase & CE8 & Pectin & 3 & 2 & 2 \\
\hline & CE12 & Pectin acetylesterase & CE12 & Pectin & 3 & 2 & 2 \\
\hline & CE16 & Acetylesterase & CE16 & Pectin; xylan; arabinoxylan & 2 & 0 & 0 \\
\hline & CE1 & Acetyl xylan esterase & CE1 & Xylan; arabinoxylan & 2 & 1 & 0 \\
\hline & & & CE1, CBM1 & Xylan; arabinoxylan & 2 & 0 & 0 \\
\hline & CE5 & Acetyl xylan esterase & CE5 & Xylan; arabinoxylan & 2 & 0 & 0 \\
\hline & CE2 & Acetyl xylan esterase & CE2 & & 1 & 0 & 0 \\
\hline & CE3 & Acetyl xylan esterase & CE3 & & $1(1)$ & $1(1)$ & $1(1)$ \\
\hline & CE4 & Acetyl xylan esterase & CE4 & & $1(1)$ & 0 & 0 \\
\hline & CE10 & Arylesterase & CE10 & & 0 & 1 & 0 \\
\hline & CE15 & 4-O-methyl-glucuronoyl methylesterase & CBM1, CE15 & & 1 & 1 & 1 \\
\hline \multirow[t]{42}{*}{ GHs } & GH1 & $\beta$-Glucosidase & GH1 & Cellulose & $1(1)$ & 0 & 0 \\
\hline & $\mathrm{GH} 3$ & $\beta$-Glucosidase & $\mathrm{GH} 3$ & Cellulose & $4(1)$ & 1 & 1 \\
\hline & GH5 & Chitosanase & GH5 & Cellulose & 3 & 2 & 1 \\
\hline & & & GH5, CBM1 & Cellulose & 1 & 0 & 0 \\
\hline & & & CBM1, GH5 & Cellulose & 1 & 0 & 0 \\
\hline & & & CBM1,GH5, CBM46 & Cellulose & 1 & 0 & 0 \\
\hline & GH6 & Endoglucanase & GH6 & Cellulose & 2 & 2 & 2 \\
\hline & & & CBM1, GH6 & Cellulose & 1 & 0 & 0 \\
\hline & GH7 & Endo- $\beta$-1,4-glucanase & GH7 & Cellulose & 2 & 2 & 2 \\
\hline & & & GH7, CBM1 & Cellulose & 2 & 0 & 0 \\
\hline & GH45 & Endoglucanase & GH45 & Cellulose & 1 & 1 & 1 \\
\hline & GH28 & Polygalacturonase & $\mathrm{GH} 28$ & Pectin & 5 & 5 & 3 \\
\hline & GH78 & $\alpha$-L-rhamnosidase & GH78 & Pectin & 0 & 1 & 0 \\
\hline & GH93 & Exo- $\alpha-\mathrm{L}-1,5$-arabinanase & GH93 & Pectin & 1 & 0 & 0 \\
\hline & GH105 & Rhamnogalacturonyl hydrolase & GH105 & Pectin & $2(1)$ & $2(1)$ & $2(1)$ \\
\hline & GH10 & Endo-1,4- $\beta$-xylanase & GH10 & Xylan; arabinoxylan & 0 & 1 & 0 \\
\hline & & & GH10, CBM1 & Xylan; arabinoxylan & 1 & 0 & 0 \\
\hline & GH11 & Xylanase & GH11 & Xylan; arabinoxylan & 2 & $1(1)$ & 0 \\
\hline & GH43 & $\beta$-Xylosidase & GH43 & Xylan; arabinoxylan & 4 & 4 & 4 \\
\hline & & & GH43, CBM35 & Xylan; arabinoxylan & 1 & 0 & 0 \\
\hline & & & GH43, GH43 & Xylan; arabinoxylan & 1 & 0 & 0 \\
\hline & GH51 & $\alpha$-L-arabinofuranosidase & GH51 & Xylan; arabinoxylan & 1 & 1 & 1 \\
\hline & GH67 & $\alpha$-Glucuronidase & GH67 & Xylan; arabinoxylan & 0 & 1 & 0 \\
\hline & GH31 & $\alpha$-Glucosidase & GH31 & Xyloglucan & 1 & 0 & 0 \\
\hline & GH35 & $\beta$-Galactosidase & GH35 & Xyloglucan & 0 & 1 & 0 \\
\hline & GH74 & Endoglucanase & GH74, CBM1 & Xyloglucan & 1 & 0 & 0 \\
\hline & GH16 & Xyloglucan:xyloglucosyl-transferase & GH16 & $\beta$-1,3-Glucan & $4(2)$ & 1 & 0 \\
\hline & GH17 & Glucan endo- $1,3-\beta$-glucosidase & GH17 & $\beta$-1,3-Glucan & $2(1)$ & 0 & 0 \\
\hline & GH55 & Exo- $\beta$-1,3-glucanase & GH55 & $\beta$-1,3-Glucan & 1 & 0 & 0 \\
\hline & GH64 & $\beta$-1,3-Glucanase & GH64 & $\beta$-1,3-Glucan & 0 & 1 & 0 \\
\hline & GH72 & $\beta$-1,3-Glucanosyl-transglycosylase & GH72 & $\beta$-1,3-Glucan & $1(1)$ & $1(1)$ & 0 \\
\hline & & & GH72, CBM43 & $\beta$-1,3-Glucan & $1(1)$ & $1(1)$ & $1(1)$ \\
\hline & GH128 & $\beta$-1,3-Glucanase & GH128 & $\beta$-1,3-Glucan & 1 & 1 & 1 \\
\hline & GH26 & $\beta$-Mannanase & CBM35, GH26 & Galactomannan & 1 & 0 & 0 \\
\hline & $\mathrm{GH} 27$ & $\alpha$-Galactosidase & $\mathrm{GH} 27$ & Galactomannan & 1 & 1 & 1 \\
\hline & GH15 & Glucoamylase & GH15, CBM20 & Starch & $3(1)$ & 2 & 2 \\
\hline & GH18 & Chitinase & GH18 & & $3(1)$ & 2 & 2 \\
\hline & GH76 & $\alpha-1,6-$ Mannanase & GH76 & & 1 & 0 & 0 \\
\hline & GH81 & Endo- $\beta$-1,3-glucanase & GH81 & & $1(1)$ & 0 & 0 \\
\hline & GH109 & $\alpha-N$-acetyl-galactosaminidase & GH109 & & $1(1)$ & 0 & 0 \\
\hline & GH114 & Endo- $\alpha$-1,4-polygalactosaminidase & GH114 & & $1(1)$ & $1(1)$ & $1(1)$ \\
\hline & GH132 & Transglycosylation & GH132 & & 0 & 1 & 0 \\
\hline \multirow[t]{5}{*}{ PLs } & PL1 & Pectate lyase & PL1 & Pectin & 6 & 5 & 4 \\
\hline & & & CBM1, PL1 & Pectin & 1 & 0 & 0 \\
\hline & PL3 & Pectate lyase & PL3 & Pectin & $4(1)$ & $5(1)$ & $4(1)$ \\
\hline & PL4 & Rhamnogalacturonan lyase & PL4 & Pectin & 1 & 2 & 1 \\
\hline & PL9 & Pectate lyase & PL9 & Pectin & 1 & 0 & 0 \\
\hline
\end{tabular}

a Statistical and functional analysis of the CAZymes that regulated by VdSec22 and VdSso1 in cotton tissue-containing medium. AAs = auxiliary activities, CBMs = carbohydrate-binding modules, CEs = carbohydrate esterases, GHs = glycoside hydrolases, and PLs = polysaccharide lyases. The family of CAZymes and their possible subtract were determined by CAZy database. The right three columns represent the number of proteins regulated by $\mathrm{VdSec} 22$, VdSso1, and common regulation in $\mathrm{VdSec} 22$ and $\mathrm{VdSso1}$, the numbers within parentheses represent the proteins not defined as typically secreted proteins. 
components of the secretome of $V$. dahliae could be further compromised and result in reduced virulence, similar to that described for related mutants in other fungal systems. In F. graminearum, FgVam7 modulates components of the secretome, including the levels of deoxynivalenol production, that play an important role in virulence (H. Zhang et al. 2016). Thus, these results support similar roles for $\mathrm{VdSec} 22$ and VdSso1, investigated herein, which affect pathogenicity of $V$. dahliae.

The vesicle-fusion model in yeast (Jahn et al. 2003) has established that the ortholog of $V d S e c 22$ mediates the vesicle fusion and trafficking between ER and trans-Golgi network. $V d S s o 1$ in $V$. dahliae is involved in the secreted vesicle fusion and trafficking to the extracellular sites. In yeast, Sec22p is a v-SNARE belonging to the ER to Golgi SNAREs complex that is involved in the transport between the ER and Golgi (Flanagan et al. 2015; Newman et al. 1990) and Ssolp is a t-SNARE belonging to the Golgi to PM SNARES complex that is involved in fusion of secretory vesicles at the PM (Aalto et al. 1993; Zhang and Shin 2006). Several studies have shown that both play important roles to mediate protein export to the extracellular sites (Duran et al. 2010; El-Kasmi et al. 2011; Kwon et al. 2014; Li et al. 2015; Toikkanen et al. 2004). For example, downregulation of SsoA, a $S$. cerevisiae Ssol homolog, resulted in a mutant phenotype characterized by accumulated secretory vesicles in the cytosol (Kwon et al. 2014). Our study confirmed that $\mathrm{VdSec} 22$ and VdSso1 are involved in transporting proteins extracellularly, since the abundance of many secreted proteins was significantly reduced after deletion of $V d S e c 22$ or VdSsol in $V$. dahliae.

Of the VdSec22- or VdSso1-regulated proteins, more than half contain a typical signal peptide regulating transport by the conventional secretory pathway. This indicates that, like their
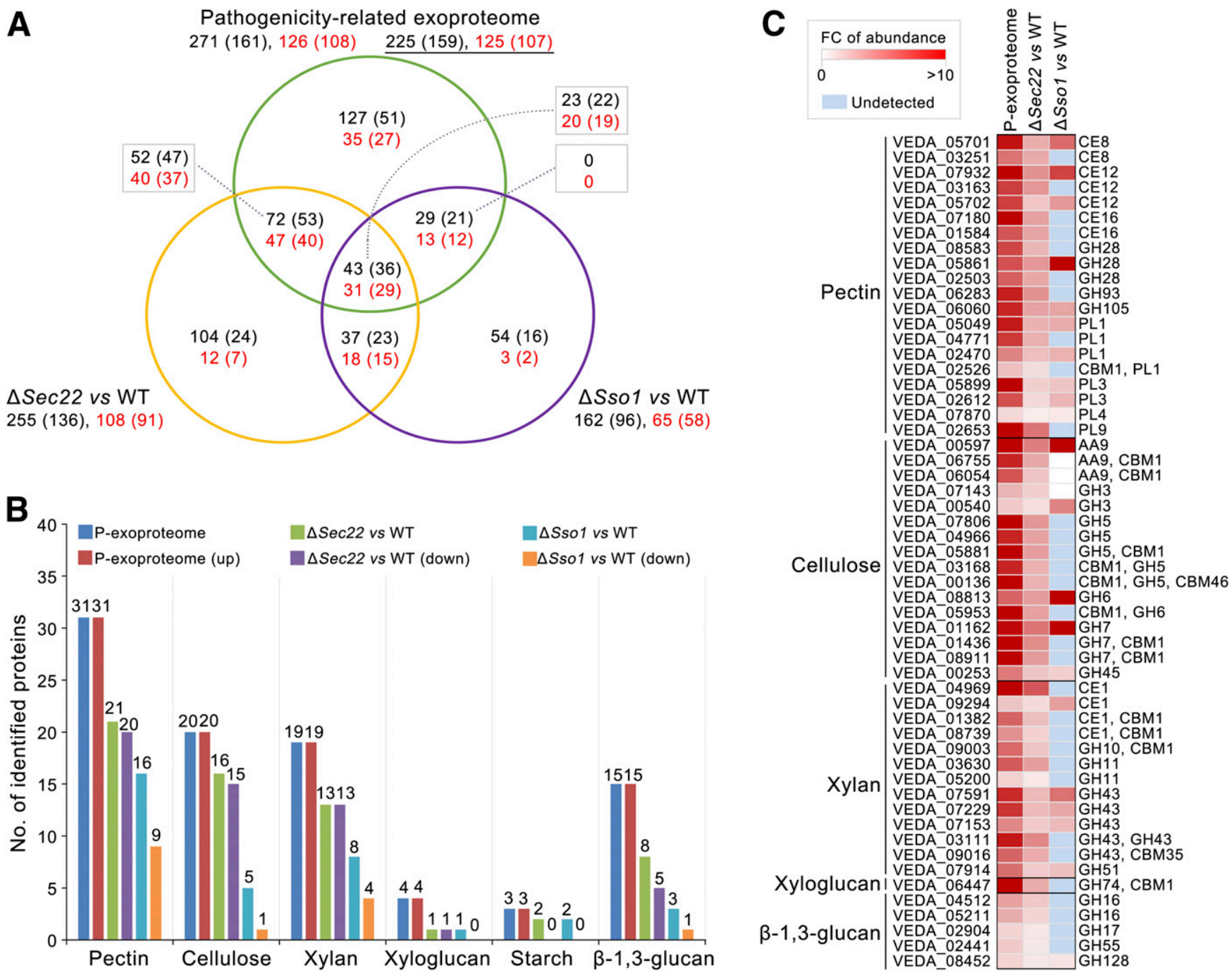

Fig. 6. TheVdSec22- and VdSso1-regulated and pathogenesis-related exoproteomes in Verticillium dahliae are enriched in plant cell wall-degrading enzymes. A, Statistical analysis of the relationship of the VdSec22- and VdSso1-regulated exoproteomes to the pathogenesis-related exoproteome. The pathogenicityrelated exoproteome was characterized previously and confers virulence on cotton leaves (Chen et al. 2016). Numbers in black represent the corresponding total protein analysis, and numbers in red represent the corresponding statistics of carbohydrate-active enzymes, while the numbers in brackets represent the typical secreted proteins. Underlined numbers represent the proteins significantly upregulated in $V$. dahliae after culture in cotton tissue-containing medium compared with the control after culture in Czapek's medium (Chen et al. 2016). Numbers in rectangles represent the proteins significantly upregulated in cotton tissue-containing medium but significantly downregulated in one or both the VdSec22 and VdSsol mutants. B, Analysis of the VdSec22-and VdSso1-regulated exoproteomes that are involved in plant cell-wall degradation. P-exoproteome represents the pathogenicity-related exoproteome, P-exoproteome (up) represents the proteins significantly upregulated in $V$. dahliae after culture in cotton tissue-containing medium compared with the control of Czapek's medium, and $\Delta S e c 22$ vs. WT (Down) and $\Delta S s o 1$ vs. WT (Down) represent the proteins significantly downregulated after deletion of VdSec 22 or VdSsol compared with the wild-type (WT) strain in cotton tissue-containing medium. C, The protein abundance fold change (FC) of the plant cell-wall degradation enzymes in P-exoproteome and the VdSec22- and VdSso1-regulated exoproteomes. 
orthologs in other fungi, VdSec22 regulates an ER to Golgi SNAREs complex and a key function of VdSsol involves fusion of secretory vesicles at the PM. Interestingly, the VdSec22- or $V d S s o 1-r e g u l a t e d$ proteins included an abundance of enzymes that have roles in plant cell-wall degradation and have, also, been shown to play crucial roles in pathogenicity (Chen et al. 2016; Gui et al. 2017; Zhang et al. 2017b). Yet this study revealed that proteins without typical secretory signals are also regulated by $\mathrm{VdSec} 22$ and VdSso1. As in M. oryzae, the virulence factors, including signal peptide-containing proteins or signal-less cytoplasmic proteins, can be secreted by a different type of pathway involving an exocyst complex (Giraldo et al. 2013). Taken together, our results suggested that $V$. dahliae employs secretory pathways both dependent and independent on VdSec 22 or VdSso1 function, to transport virulence factors to extracellular sites.

In conclusion, this study characterized the role of two key vesicle-fusion components, $V d S e c 22$ or $V d S s o 1$, in exoproteome production in $V$. dahliae $\mathrm{Vd} 991$ and provided evidence that these vesicle-fusion components play an important role in virulence. Characterization of the VdSec22- or VdSso1-mediated exoproteome proteins suggested that a number of these proteins are involved in plant cell-wall degradation and are the likely mechanism by which virulence is compromised in the
$V d S e c 22$ or $V d S s o l$ mutants. The work confirms that V. dahliae employs more than a single secretory route to transport proteins to extracellular sites during infection.

\section{MATERIALS AND METHODS}

\section{Identification and prediction of genes encoding vesicle-fusion components in Verticillium dahlia.}

Vesicle-fusion components in the $V$. dahliae Vd991 genome (Chen et al. 2018) were identified using BLASTN and BLASTP searches (Altschul et al. 1997), with previously characterized $V$. dahliae Vd.Ls17 vesicle-fusion component sequences (Yang et al. 2013) as queries, with the cutoff $E$ value < 1e-10. The vesicle-fusion component gene orthologs between Vd991 and yeast were assigned based on sequence similarity (Yang et al. 2013). Potential transmembrane domains were predicted with TMHMM2.0 and Phobius (Käll et al. 2004; Krogh et al. 2001). The putative cellular association of SNARE complexes, Rab proteins, and SM proteins with trafficking steps was depicted in $V$. dahliae based on the yeast model (Jahn et al. 2003). Clustal $X 2$ was used for multiple sequence alignment of the vesicle-fusion component VdVam7 to orthologs in other fungi (Larkin et al. 2007).

\begin{tabular}{|c|c|c|c|}
\hline Gene-ID & CAZymes family & Secreted & Fold chang \\
\hline VEDA_03687 & AA9 & & 6.64 \\
\hline VEDA_04614 & CBM63 & $Y$ & 2.82 \\
\hline VEDA_03908 & $\mathrm{GH} 3$ & & 8.69 \\
\hline VEDA_09734 & $\mathrm{GH} 3$ & $\mathrm{Y}$ & 4.24 \\
\hline VEDA_02783 & $\mathrm{GH} 12$ & & 1.59 \\
\hline VEDA_05769 & $\mathrm{GH} 12, \mathrm{CBM} 1$ & $Y$ & 1.81 \\
\hline VEDA_03802 & CE16 & $Y$ & 4.89 \\
\hline VEDA_01417 & $\mathrm{GH} 28$ & $Y$ & 9.63 \\
\hline VEDA_01287 & $\mathrm{GH} 78$ & Y & 7.65 \\
\hline VEDA_01449 & PL1 & Y & 5.17 \\
\hline VEDA_05925 & PL1 & $Y$ & 1.74 \\
\hline VEDA_09566 & AA3 & $Y$ & 5.22 \\
\hline VEDA_08571 & AA3 & Y & 4.54 \\
\hline VEDA_05900 & AA3 & $Y$ & 2.83 \\
\hline VEDA_04228 & AA5 & $Y$ & 1.60 \\
\hline VEDA_06395 & AA7 & $\mathrm{Y}$ & 1.82 \\
\hline VEDA_05547 & CBM20 & $Y$ & 10.88 \\
\hline VEDA_08100 & CE1 & & 1.86 \\
\hline VEDA_06795 & CE10 & & 6.11 \\
\hline VEDA_03875 & CE10 & $Y$ & 3.42 \\
\hline VEDA_07242 & CE10 & $Y$ & 3.26 \\
\hline VEDA_02801 & CE10 & $Y$ & 2.54 \\
\hline VEDA_08919 & CE5 & Y & 3.41 \\
\hline VEDA_08569 & $\mathrm{GH} 13$ & Y & 6.00 \\
\hline VEDA_01314 & $\mathrm{GH} 16$ & & 3.36 \\
\hline VEDA_06932 & $\mathrm{GH} 16$ & & 3.30 \\
\hline VEDA_07512 & $\mathrm{GH} 16$ & & 2.12 \\
\hline VEDA_02588 & $\mathrm{GH} 17$ & $Y$ & 2.36 \\
\hline VEDA_09336 & $\mathrm{GH} 37$ & $Y$ & 8.99 \\
\hline VEDA_03777 & $\mathrm{GH} 43$ & $Y$ & 3.91 \\
\hline VEDA_07148 & GH55 & $Y$ & 4.10 \\
\hline VEDA_04950 & $\mathrm{GH} 72$ & $Y$ & 2.41 \\
\hline VEDA_07224 & $\mathrm{GH} 79$ & $Y$ & 8.19 \\
\hline VEDA_04719 & GH81 & $Y$ & 5.10 \\
\hline VEDA_09719 & $\mathrm{GH} 125$ & $Y$ & 4.17 \\
\hline & & dance & 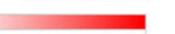 \\
\hline
\end{tabular}

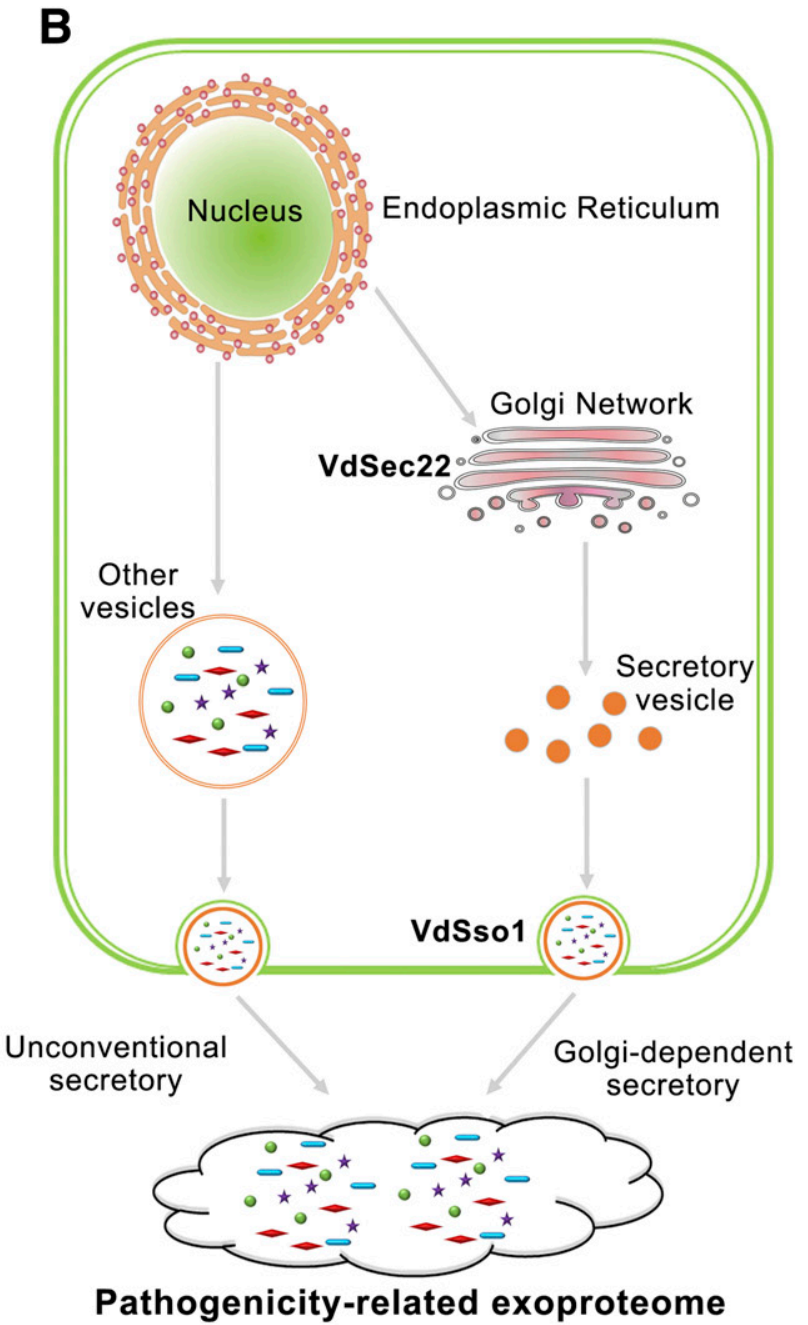

Fig. 7. Verticillium dahliae translocates the pathogenicity-related exoproteome independently of VdSec 22 and VdSso1. A, Protein abundance fold-change (FC) of the carbohydrate-active enzymes (CAZymes) regulated independently of VdSec22 and VdSso1 in strain Vd991. The protein abundances were determined from $V$. dahliae cultured in cotton tissue-containing medium compared with the basic medium (Czapek-Dox medium) (Chen et al. 2016). Y means the typical secreted proteins contain signal peptide; C, P, and X represent the functions of proteins involved in cellulose, pectin, and xyloglucan degradation, respectively. B, Schematic overview showing how $V$. dahliae translocates the pathogenicity-related exoproteome through VdSec22 and VdSso1-dependent or -independent pathways. 


\section{Fungal transformations.}

Targeted gene-deletion constructs were generated based on a previously described method (Liu et al. 2013). Briefly, approximately 1-kb flanking sequences of VdSec22 and VdSsol were amplified from $V$. dahliae Vd991 genomic DNA, and the hygromycin phosphotransferase gene cassette, including the $\operatorname{TrpC}$-promoter, hygromycin phosphotransferase gene, and Nos terminator, were amplified from the pUC-Hyg vector. The above three fragments were fused to one fragment via fusion PCR. Nested PCR was then performed to obtain an internal amplicon that was then cloned into a pGKO2-Gateway vector using a standard BP reaction (Khang et al. 2005). All primers used for vector construction are listed in Supplementary Table S8. The transformants were complementated with fragments containing the $V d S e c 22$ or $V d S s o l$ wild-type coding sequences, including sequences of the respective approximately 1-kb upstream, native promoters, and the approximately $0.5-\mathrm{kb}$ downstream regions. These were amplified from $V$. dahliae $\mathrm{Vd} 991$ genomic DNA and were cloned into the binary vector pCOM that carried geneticin resistance (Zhou et al. 2013). Homologous recombination via Agrobacterium tumefaciens-mediated transformation of $V$. dahliae was performed as described previously (Mullins and Kang 2001). Transformants were selected on potato dextrose agar (PDA) medium (per liter: $200 \mathrm{~g}$ of potato, $20 \mathrm{~g}$ of glucose, and $15 \mathrm{~g}$ of agar) with cefotaxime $(200 \mu \mathrm{g} / \mathrm{ml})$, hygromycin $(50 \mu \mathrm{g} / \mathrm{ml})$, and 5-fluoro-2'-deoxyuridine (200 $\mu \mathrm{g} / \mathrm{ml})$. Single spore isolation was performed for all transformants, followed by PCR verification with two primer pairs, i.e., a hygromycin phosphotransferase gene-specific primer pair and the internal test primer pair for $V d S e c 22$ or $V d S s o 1$, respectively.

\section{Pathogenicity assays.}

For pathogenicity assays, all strains, including $V d S e c 22$ or VdSsol targeted deletion strains and the wild-type strain Vd991, were cultured on PDA for 7 days at $25^{\circ} \mathrm{C}$, and conidial suspensions $\left(5 \times 10^{6}\right.$ conidia $\left./ \mathrm{ml}\right)$ were prepared using sterile water. Cotton (a susceptible cultivar of Gossypium hirsutum L., 'Junmian No. 1') plants were grown at $28^{\circ} \mathrm{C}$ in a greenhouse (14-h day and 10-h night cycle). For each transformant, 2-week-old cotton seedlings were inoculated with $5 \times 10^{6}$ conidia per milliliter, using a root-dip method (D. D. Zhang et al. 2016), 30 cotton seedlings were included in each treatment, and each treatment had a total of three replicates. External disease symptoms and vascular discoloration of inoculated cotton plants were observed at 21 days postinoculation. Fungal biomass in infected cotton plants was determined as previously described (Santhanam and Thomma 2013). qPCR was performed using the qPCR SYBR premix Ex Taq II kit (TaKaRa) with the primers of the cotton $18 S$ gene. Unpaired Student's $t$ tests were performed to determine statistical significance at $P<0.05$ between inoculated and uninoculated treatments groups.

\section{Growth phenotype assay.}

The radial growth phenotypes of VdSec22 and VdSsol targeted deletion strains and the wild-type strain Vd991 were assessed by placing a $2-\mu 1$ droplet of a conidial suspension of $2 \times 10^{6} \mathrm{conidia} / \mathrm{ml}$ in the center of a medium plate and incubating it at $22^{\circ} \mathrm{C}$. Media were prepared with four different carbon sources, namely (per liter), $30.0 \mathrm{~g}$ of sucrose, $10.0 \mathrm{~g}$ of pectin, $10.0 \mathrm{~g}$ of sodium carboxymethyl cellulose, and $15.0 \mathrm{~g}$ of starch, and Czapek-Dox agar (per liter: $2 \mathrm{~g}$ of $\mathrm{NaNO}_{3}, 0.5 \mathrm{~g}$ of $\mathrm{MgSO}_{4} \cdot 7 \mathrm{H}_{2} \mathrm{O}, 0.5 \mathrm{~g}$ of $\mathrm{KCl}, 100 \mathrm{mg}$ of $14 \mathrm{FeSO}_{4} \cdot 7 \mathrm{H}_{2} \mathrm{O}$, and $1 \mathrm{~g}$ of $\mathrm{K}_{2} \mathrm{HPO}_{4}, \mathrm{pH}$ 7.2). Growth phenotypes and growth rates (colony diameter) were investigated after 10 days of incubation at $25^{\circ} \mathrm{C}$.

\section{Wilting activity of the exoproteome.}

The exoproteome of VdSec22 or VdSsol targeted deletion strains and the wild-type strain Vd991 were extracted from cotton-containing medium as described previously (Chen et al. 2016). In detail, whole plants (3-week-old plants of susceptible cultivar Junmian No. 1) without leaves were harvested and were ground into powder in liquid nitrogen, and the cottoncontaining medium was prepared using basic Czapek-Dox solution supplemented with $1 \%$ (wt/vol) cotton tissue powder. To induce the plant-associated exoproteome in $V$. dahliae, $5 \mathrm{ml}$ of conidial suspensions of $5 \times 10^{6}$ conidia $/ \mathrm{ml}$ for each strain were cultured in the cotton-containing medium for 3 days. Three replicates of every preparation were incubated at $25^{\circ} \mathrm{C}$ and were centrifuged at $180 \mathrm{rpm}$. After 5 days of incubation, culture suspensions were filtered through four layers of gauze, and the fungal biomass was pelleted via centrifugation. Supernatants were collected, and the solution was filtered through a $0.22-\mu \mathrm{m}$ membrane (Millipore). The Avant purification system (GE Healthcare) was used for exoproteome enrichment; the filtered solution was enriched, using a Hitrap Capto SP/Q column (GE Healthcare), and desalted, using sephasex G75 (GE Healthcare). Finally, the purified exoproteome was lyophilized before being resuspended in sterilized water. The exoproteome concentration was determined using 2-D Quant kit assay reagents (GE Healthcare), following the manufacturer's instructions.

To determine the wilting activity of exoproteomes from $V d S e c 22$ or VdSsol targeted deletion strains, the 'Junmian No. 1 ' cotyledons were collected, including petioles, from 2-weekold seedlings and were inserted directly into a $0.5-\mathrm{ml}$ microcentrifuge tube that contained $20.0 \mu \mathrm{l}$ of exoproteome solution $(1.0 \mu \mathrm{g})$. When all the solution was absorbed by the cotyledons, $400 \mu \mathrm{l}$ of sterilized water was added to the tube, which was then placed in a box with a transparent cover, to maintain appropriate moisture. Cotyledon phenotypes were investigated $96 \mathrm{~h}$ after incubation at $25^{\circ} \mathrm{C}$; five cotyledons were placed in each tube and there were three replicates. The exoproteome of wildtype strain Vd991 and cotton-containing medium were used as positive and negative controls, respectively.

\section{iTRAQ labeling, spectrometric analysis, and data processing.}

Exoproteome samples containing $100 \mu \mathrm{g}$ of protein were digested, using Trypsin Gold (Promega), for $16 \mathrm{~h}$ at $37^{\circ} \mathrm{C}$, to generate peptides. Peptides were then dried by vacuum centrifugation and were resuspended in $0.5 \mathrm{M}$ triethylammonium bicarbonate. Desalted peptides were then labeled with iTRAQ reagents (Applied Biosystems), following the manufacturer's instructions (Zieske 2006). Three replicates of extracted proteins from the $V d S e c 22$ deletion strain grown in cottoncontaining medium were labeled with reagents 117 and 118, while extracted proteins of $V d S s o l$ deletion strain were labeled with reagents 119 and 121, and extracted proteins of wild-type strain Vd991 were labeled with reagents 115 and 116. The peptide mixtures were pooled and dried by vacuum centrifugation. These pooled iTRAQ-labeled peptide mixtures were fractionated into 10 portions, using strong cationic exchange chromatography, and were desalted in a nanobored C18 column with a picofrit nanospray tip $(365 \mu \mathrm{m}$ i.d $\times 15 \mathrm{~cm}, 5 \mu \mathrm{m}$ particles, 15-nm pore size) (Phenomenex Inc.).

Data acquisition was performed using time-of-flight mass spectrometry (TOF-MS) in a TripleTOF5600 MS system (AB Sciex) fitted with a Nanospray III source (AB Sciex) and using a pulled quartz tip as the emitter (New Objectives), as described previously (Chen et al. 2016). Raw files were processed using the Mascot computational proteomics platform version 2.3.01. Fragmentation spectra were searched against the Vd991 protein-coding genome sequences (Chen et al. 2018) with 
fragment mass tolerance set to $20 \mathrm{mmu}$ and with up to one missed cleavage. For database searches, carabamidomethlyation of cysteine was set as a fixed modification, while the oxidation of glutamine and protein $\mathrm{N}$-terminal acetylation were chosen as variable modifications. Both peptide and protein identifications were filtered at $1 \%$ false discovery rates, so proteins identified with at least two unique peptides were independent of peptide score.

\section{Exoproteome annotation.}

Functional annotations of differentially abundant proteins were performed using the GO Consortium database, and the WEGO (Web Gene Ontology Annotation Plot) software was used to retrieve GO annotations of unigenes for describing GO catalytic activity class (Ye et al. 2006). The significant differences of GO enrichment were analyzed by hypergeometric test for each GO term and were subsequently corrected for multiple testing errors, with $401 \mathrm{GO}$ annotations, 280 enriched in GO: 0003824 catalytic activity, and 156 enriched in GO:0016787 hydrolase activity of all the identified proteins. Proteins that were localized only extracellularly (WoLF PSORT software) (Horton et al. 2007) and contained a signal peptide and a signal peptide cleavage site (SignalP 4.1 software) (Petersen et al. 2011) but lacked transmembrane domains (TMHMM 2.0 and Phobius software) (Krogh et al. 2001) were defined as secreted proteins. Proteins less than 400 amino acids in length and containing up to four cysteine residues were designated SCRPs. Annotation of putative CAZymes was performed using the hidden Markov model-based routine of the CAZymes database (Cantarel et al. 2009). Significant hits compared with this database were identified using the set of putative CAZymes from BLAST searches $(E$ value $<1 \mathrm{e}-5$, similarity $>30 \%)$ (Altschul et al. 1997), which were subsequently used for annotation. CAZymes involved in plant cell-wall degradation were then classified, using methods outlined in previous studies (Battaglia et al. 2011; Goodwin et al. 2011). Homologs of known pathogenicity-related genes were predicted using the PHI-base database (version 3.6) (Winnenburg et al. 2008).

\section{LITERATURE CITED}

Aalto, M. K., Ronne, H., and Keränen, S. 1993. Yeast syntaxins Sso1p and Sso $2 p$ belong to a family of related membrane proteins that function in vesicular transport. EMBO J. 12:4095-4104.

Altschul, S. F., Madden, T. L., Schäffer, A. A., Zhang, J., Zhang, Z., Miller, W., and Lipman, D. J. 1997. Gapped BLAST and PSI-BLAST: A new generation of protein database search programs. Nucleic Acids Res. 25:3389-3402.

Battaglia, E., Benoit, I., van den Brink, J., Wiebenga, A., Coutinho, P. M., Henrissat, B., and de Vries, R. P. 2011. Carbohydrate-active enzymes from the zygomycete fungus Rhizopus oryzae: A highly specialized approach to carbohydrate degradation depicted at genome level. BMC Genomics 12:38.

Bennett, M. K. 1995. SNAREs and the specificity of transport vesicle targeting. Curr. Opin. Cell Biol. 7:581-586.

Bernardo, S. M., Rane, H. S., Chavez-Dozal, A., and Lee, S. A. 2014. Secretion and filamentation are mediated by the Candida albicans t-SNAREs Sso2p and Sec9p. FEMS Yeast Res. 14:762-775.

Bonifacino, J. S., and Glick, B. S. 2004. The mechanisms of vesicle budding and fusion. Cell 116:153-166.

Burri, L., and Lithgow, T. 2004. A complete set of SNAREs in yeast. Traffic 5:45-52.

Cai, H., Reinisch, K., and Ferro-Novick, S. 2007. Coats, tethers, Rabs, and SNAREs work together to mediate the intracellular destination of a transport vesicle. Dev. Cell 12:671-682.

Cantarel, B. L., Coutinho, P. M., Rancurel, C., Bernard, T., Lombard, V., and Henrissat, B. 2009. The Carbohydrate-Active EnZymes database (CAZy): An expert resource for glycogenomics. Nucleic Acids Res. 37:D233-D238.

Chen, J. Y., Liu, C., Gui, Y. J., Si, K. W., Zhang, D. D., Wang, J., Short, D. P. G., Huang, J. Q., Li, N. Y., Liang, Y., Zhang, W. Q., Yang, L., Ma, X. F., Li, T. G., Zhou, L., Wang, B. L., Bao, Y. M., Subbarao, K. V., Zhang, G. Y., and Dai, X. F. 2018. Comparative genomics reveals cotton-specific virulence factors in flexible genomic regions in Verticillium dahliae and evidence of horizontal gene transfer from Fusarium. New Phytol. 217:756-770.

Chen, J. Y., Xiao, H. L., Gui, Y. J., Zhang, D. D., Li, L., Bao, Y. M., and Dai, X. F. 2016. Characterization of the Verticillium dahliae exoproteome involves in pathogenicity from cotton-containing medium. Front. Microbiol. 7:1709.

Chu, J., Li, W. F., Cheng, W., Lu, M., Zhou, K. H., Zhu, H. Q., Li, F. G., and Zhou, C. Z. 2015. Comparative analyses of secreted proteins from the phytopathogenic fungus Verticillium dahliae in response to nitrogen starvation. Biochim. Biophys. Acta 1854:437-448.

de Jonge, R., Bolton, M. D., and Thomma, B. P. 2011. How filamentous pathogens co-opt plants: The ins and outs of fungal effectors. Curr. Opin. Plant Biol. 14:400-406.

de Jonge, R., van Esse, H. P., Maruthachalam, K., Bolton, M. D., Santhanam, P., Saber, M. K., Zhang, Z., Usami, T., Lievens, B., Subbarao, K. V., and Thomma, B. P. 2012. Tomato immune receptor Ve1 recognizes effector of multiple fungal pathogens uncovered by genome and RNA sequencing. Proc. Natl. Acad. Sci. U.S.A. 109:5110-5115.

De Marchis, F., Bellucci, M., and Pompa, A. 2013. Unconventional pathways of secretory plant proteins from the endoplasmic reticulum to the vacuole bypassing the Golgi complex. Plant Signal. Behav. 8:e25129.

de Sain, M., and Rep, M. 2015. The role of pathogen-secreted proteins in fungal vascular wilt diseases. Int. J. Mol. Sci. 16:23970-23993.

Dou, X., Wang, Q., Qi, Z., Song, W., Wang, W., Guo, M., Zhang, H., Zhang, Z., Wang, P., and Zheng, X. 2011. MoVam7, a conserved SNARE involved in vacuole assembly, is required for growth, endocytosis, ROS accumulation, and pathogenesis of Magnaporthe oryzae. PLoS One 6:e16439.

Drakakaki, G., and Dandekar, A. 2013. Protein secretion: How many secretory routes does a plant cell have? Plant Sci. 203-204:74-78.

Duran, J. M., Anjard, C., Stefan, C., Loomis, W. F., and Malhotra, V. 2010. Unconventional secretion of Acb1 is mediated by autophagosomes. J. Cell Biol. 188:527-536.

El-Kasmi, F., Pacher, T., Strompen, G., Stierhof, Y. D., Müller, L. M., Koncz, C., Mayer, U., and Jürgens, G. 2011. Arabidopsis SNARE protein SEC22 is essential for gametophyte development and maintenance of Golgi-stack integrity. Plant J. 66:268-279.

Fasshauer, D., Sutton, R. B., Brunger, A. T., and Jahn, R. 1998. Conserved structural features of the synaptic fusion complex: SNARE proteins reclassified as Q- and R-SNAREs. Proc. Natl. Acad. Sci. U.S.A. 95:15781-15786.

Flanagan, J. J., Mukherjee, I., and Barlowe, C. 2015. Examination of Sec22 homodimer formation and role in SNARE-dependent membrane fusion. J. Biol. Chem. 290:10657-10666.

Franceschetti, M., Maqbool, A., Jiménez-Dalmaroni, M. J., Pennington, H. G., Kamoun, S., and Banfield, M. J. 2017. Effectors of filamentous plant pathogens: Commonalities amid diversity. Microbiol. Mol. Biol. Rev. 81:e0066-e16.

Giraldo, M. C., Dagdas, Y. F., Gupta, Y. K., Mentlak, T. A., Yi, M., Martinez-Rocha, A. L., Saitoh, H., Terauchi, R., Talbot, N. J., and Valent, B. 2013. Two distinct secretion systems facilitate tissue invasion by the rice blast fungus Magnaporthe oryzae. Nat. Commun. 4:1996.

Goodwin, S. B., M'barek, S. B., Dhillon, B., Wittenberg, A. H., Crane, C. F., Hane, J. K., Foster, A. J., Van der Lee, T. A., Grimwood, J., Aerts, A., Antoniw, J., Bailey, A., Bluhm, B., Bowler, J., Bristow, J., van der Burgt, A., Canto-Canché, B., Churchill, A. C., Conde-Ferràez, L., Cools, H. J., Coutinho, P. M., Csukai, M., Dehal, P., De Wit, P., Donzelli, B., van de Geest, H. C., van Ham, R. C., Hammond-Kosack, K. E., Henrissat, B., Kilian, A., Kobayashi, A. K., Koopmann, E., Kourmpetis, Y., Kuzniar, A., Lindquist, E., Lombard, V., Maliepaard, C., Martins, N., Mehrabi, R., Nap, J. P., Ponomarenko, A., Rudd, J. J., Salamov, A., Schmutz, J., Schouten, H. J., Shapiro, H., Stergiopoulos, I., Torriani, S. F., Tu, H., de Vries, R. P., Waalwijk, C., Ware, S. B., Wiebenga, A., Zwiers, L. H., Oliver, R. P., Grigoriev, I. V., and Kema, G. H. 2011. Finished genome of the fungal wheat pathogen Mycosphaerella graminicola reveals dispensome structure, chromosome plasticity, and stealth pathogenesis. PLoS Genet. 7:e1002070.

Gui, Y. J., Chen, J. Y., Zhang, D. D., Li, N. Y., Li, T. G., Zhang, W. Q., Wang, X. Y., Short, D. P. G., Li, L., Guo, W., Kong, Z. Q., Bao, Y. M., Subbarao, K. V., and Dai, X. F. 2017. Verticillium dahliae manipulates plant immunity by glycoside hydrolase 12 proteins in conjunction with carbohydrate-binding module 1. Environ. Microbiol. 19:1914-1932.

Hong, W. 2005. SNAREs and traffic. Biochim. Biophys. Acta 1744: 493-517.

Horton, P., Park, K. J., Obayashi, T., Fujita, N., Harada, H., Adams-Collier, C. J., and Nakai, K. 2007. WoLF PSORT: Protein localization predictor. Nucleic Acids Res. 35:W585-W587.

Irieda, H., Maeda, H., Akiyama, K., Hagiwara, A., Saitoh, H., Uemura, A., Terauchi, R., and Takano, Y. 2014. Colletotrichum orbiculare secretes virulence effectors to a biotrophic interface at the primary hyphal neck via exocytosis coupled with Sec22-mediated traffic. Plant Cell 26:2265-2281. 
Jahn, R., Lang, T., and Südhof, T. C. 2003. Membrane fusion. Cell 112 : 519-533.

Käll, L., Krogh, A., and Sonnhammer, E. L. 2004. A combined transmembrane topology and signal peptide prediction method. J. Mol. Biol. 338:1027-1036.

Khang, C. H., Park, S. Y., Lee, Y. H., and Kang, S. 2005. A dual selection based, targeted gene replacement tool for Magnaporthe grisea and Fusarium oxysporum. Fungal Genet. Biol. 42:483-492.

Kienle, N., Kloepper, T. H., and Fasshauer, D. 2009. Phylogeny of the SNARE vesicle fusion machinery yields insights into the conservation of the secretory pathway in fungi. BMC Evol. Biol. 9:19.

Klosterman, S. J., Atallah, Z. K., Vallad, G. E., and Subbarao, K. V. 2009 Diversity, pathogenicity, and management of Verticillium species. Annu. Rev. Phytopathol. 47:39-62.

Klosterman, S. J., Subbarao, K. V., Kang, S., Veronese, P., Gold, S. E., Thomma, B. P., Chen, Z., Henrissat, B., Lee, Y. H., Park, J., GarciaPedrajas, M. D., Barbara, D. J., Anchieta, A., de Jonge, R., Santhanam, P., Maruthachalam, K., Atallah, Z., Amyotte, S. G., Paz, Z., Inderbitzin, P., Hayes, R. J., Heiman, D. I., Young, S., Zeng, Q., Engels, R., Galagan, J., Cuomo, C. A., Dobinson, K. F., and Ma, L. J. 2011. Comparative genomics yields insights into niche adaptation of plant vascular wilt pathogens. PLoS Pathog. 7:e1002137.

Kombrink, A., Rovenich, H., Shi-Kunne, X., Rojas-Padilla, E., van den Berg, G. C., Domazakis, E., de Jonge, R., Valkenburg, D. J., SánchezVallet, A., Seidl, M. F., and Thomma, B. P. 2017. Verticillium dahliae LysM effectors differentially contribute to virulence on plant hosts. Mol. Plant Pathol. 18:596-608.

Koumandou, V. L., Dacks, J. B., Coulson, R. M., and Field, M. C. 2007. Control systems for membrane fusion in the ancestral eukaryote; evolution of tethering complexes and SM proteins. BMC Evol. Biol. 7:29.

Krogh, A., Larsson, B., von Heijne, G., and Sonnhammer, E. L. 2001. Predicting transmembrane protein topology with a hidden Markov model: Application to complete genomes. J. Mol. Biol. 305:567-580.

Kwon, M. J., Arentshorst, M., Fiedler, M., de Groen, F. L., Punt, P. J., Meyer, V., and Ram, A. F. 2014. Molecular genetic analysis of vesicular transport in Aspergillus niger reveals partial conservation of the molecular mechanism of exocytosis in fungi. Microbiology 160:316-329.

Larkin, M. A., Blackshields, G., Brown, N. P., Chenna, R., McGettigan, P. A., McWilliam, H., Valentin, F., Wallace, I. M., Wilm, A., Lopez, R., Thompson, J. D., Gibson, T. J., and Higgins, D. G. 2007. Clustal W and Clustal X version 2.0. Bioinformatics 23:2947-2948.

Li, B., Liu, L., Li, Y., Dong, X., Zhang, H., Chen, H., Zheng, X., and Zhang, Z. 2017. The FgVps39-FgVam7-FgSso1 complex mediates vesicle trafficking and is important for the development and virulence of Fusarium graminearum. Mol. Plant-Microbe Interact. 30:410-422.

Li, X., Wu, Y., Shen, C., Belenkaya, T. Y., Ray, L., and Lin, X. 2015. Drosophila p24 and Sec22 regulate Wingless trafficking in the early secretory pathway. Biochem. Biophys. Res. Commun. 463:483-489.

Lipka, V., Kwon, C., and Panstruga, R. 2007. SNARE-ware: The role of SNARE-domain proteins in plant biology. Annu. Rev. Cell Dev. Biol. 23: 147-174.

Liu, S. Y., Chen, J. Y., Wang, J. L., Li, L., Xiao, H. L., Adam, S. M., and Dai, X. F. 2013. Molecular characterization and functional analysis of a specific secreted protein from highly virulent defoliating Verticillium dahliae. Gene 529:307-316.

Liu, T., Song, T., Zhang, X., Yuan, H., Su, L., Li, W., Xu, J., Liu, S., Chen, L., Chen, T., Zhang, M., Gu, L., Zhang, B., and Dou, D. 2014. Unconventionally secreted effectors of two filamentous pathogens target plant salicylate biosynthesis. Nat. Commun. 5:4686.

Mullins, E. D., and Kang, S. 2001. Transformation: A tool for studying fungal pathogens of plants. Cell. Mol. Life Sci. 58:2043-2052.

Newman, A. P., Shim, J., and Ferro-Novick, S. 1990. BET1, BOS1, and SEC22 are members of a group of interacting yeast genes required for transport from the endoplasmic reticulum to the Golgi complex. Mol. Cell. Biol. 10:3405-3414.

Ostrowicz, C. W., Meiringer, C. T., and Ungermann, C. 2008. Yeast vacuole fusion: A model system for eukaryotic endomembrane dynamics. Autophagy 4:5-19.

Palade, G. 1975. Intracellular aspects of the process of protein synthesis. Science 189:867.

Pelham, H. R. 2001. SNAREs and the specificity of membrane fusion. Trends Cell Biol. 11:99-101.

Petersen, T. N., Brunak, S., von Heijne, G., and Nielsen, H. 2011. SignalP 4.0: Discriminating signal peptides from transmembrane regions. Nat. Methods 8:785-786.

Qi, Z., Liu, M., Dong, Y., Zhu, Q., Li, L., Li, B., Yang, J., Li, Y., Ru, Y., Zhang, H., Zheng, X., Wang, P., and Zhang, Z. 2016. The syntaxin protein (MoSyn8) mediates intracellular trafficking to regulate conidiogenesis and pathogenicity of rice blast fungus. New Phytol. 209:1655-1667.

Šamaj, J., Müller, J., Beck, M., Böhm, N., and Menzel, D. 2006. Vesicular trafficking, cytoskeleton and signalling in root hairs and pollen tubes. Trends Plant Sci. 11:594-600.

Sanderfoot, A. 2007. Increases in the number of SNARE genes parallels the rise of multicellularity among the green plants. Plant Physiol. 144:6-17

Santhanam, P., and Thomma, B. P. 2013. Verticillium dahliae Sge1 differentially regulates expression of candidate effector genes. Mol. Plant-Microbe Interact. 26:249-256.

Schekman, R. 2010. Charting the secretory pathway in a simple eukaryote. Mol. Biol. Cell 21:3781-3784.

Song, W., Dou, X., Qi, Z., Wang, Q., Zhang, X., Zhang, H., Guo, M., Dong, S., Zhang, Z., Wang, P., and Zheng, X. 2010. R-SNARE homolog MoSec22 is required for conidiogenesis, cell wall integrity, and pathogenesis of Magnaporthe oryzae. PLoS One 5:e13193.

Tian, L., Xu, R., Wang, Z., Liu, N., Feng, F., and Qu, Z. 2015. [Vesicular transport protein $\mathrm{VdSec} 22$ is involved in secretion of extracellular protein and pathogenicity in Verticillium dahliae.]. Wei Sheng Wu Xue Bao 55:873-881.

Toikkanen, J. H., Sundqvist, L., and Keränen, S. 2004. Kluyveromyces lactis SSOI and SEB1 genes are functional in Saccharomyces cerevisiae and enhance production of secreted proteins when overexpressed. Yeast 21:1045-1055.

Ungar, D., and Hughson, F. M. 2003. SNARE protein structure and function. Annu. Rev. Cell Dev. Biol. 19:493-517.

Wada, Y., Nakamura, N., Ohsumi, Y., and Hirata, A. 1997. Vam3p, a new member of syntaxin related protein, is required for vacuolar assembly in the yeast Saccharomyces cerevisiae. J. Cell Sci. 110:1299-1306.

Wickner, W. 2010. Membrane fusion: Five lipids, four SNAREs, three chaperones, two nucleotides, and a Rab, all dancing in a ring on yeast vacuoles. Annu. Rev. Cell Dev. Biol. 26:115-136.

Winnenburg, R., Urban, M., Beacham, A., Baldwin, T. K., Holland, S. Lindeberg, M., Hansen, H., Rawlings, C., Hammond-Kosack, K. E., and Köhler, J. 2008. PHI-base update: Additions to the pathogen host interaction database. Nucleic Acids Res. 36:D572-D576.

Yang, X., Ben, S., Sun, Y., Fan, X., Tian, C., and Wang, Y. 2013. Genomewide identification, phylogeny and expression profile of vesicle fusion components in Verticillium dahliae. PLoS One 8:e68681.

Yao, H. Y., and Xue, H. W. 2011. Signals and mechanisms affecting vesicular trafficking during root growth. Curr. Opin. Plant Biol. 14: 571-579.

Ye, J., Fang, L., Zheng, H., Zhang, Y., Chen, J., Zhang, Z., Wang, J., Li, S., Li, R., Bolund, L., and Wang, J. 2006. WEGO: A web tool for plotting GO annotations. Nucleic Acids Res. 34:W293-W297.

Zhang, D. D., Wang, X. Y., Chen, J. Y., Kong, Z. Q., Gui, Y. J., Li, N. Y., Bao, Y. M., and Dai, X. F. 2016. Identification and characterization of a pathogenicity-related gene VdCYP1 from Verticillium dahliae. Sci. Rep. 6:27979.

Zhang, H., Li, B., Fang, Q., Li, Y., Zheng, X., and Zhang, Z. 2016. SNARE protein FgVam7 controls growth, asexual and sexual development, and plant infection in Fusarium graminearum. Mol. Plant Pathol. 17:108-119.

Zhang, L., Ni, H., Du, X., Wang, S., Ma, X. W., Nürnberger, T., Guo, H. S., and Hua, C. 2017a. The Verticillium-specific protein VdSCP7 localizes to the plant nucleus and modulates immunity to fungal infections. New Phytol. 215:368-381.

Zhang, W. Q., Gui, Y. J., Short, D. P. G., Li, T. G., Zhang, D. D., Zhou, L., Liu, C., Bao, Y. M., Subbarao, K. V., Chen, J. Y., and Dai, X. F. 2017b. Verticillium dahliae transcription factor VdFTF1 regulates the expression of multiple secreted virulence factors and is required for full virulence in cotton. Mol. Plant Pathol. 19:841-857.

Zhang, Y., and Shin, Y. K. 2006. Transmembrane organization of yeast syntaxin-analogue Sso1p. Biochemistry 45:4173-4181.

Zhao, W., Dong, S., Ye, W., Hua, C., Meijer, H. J., Dou, X., Govers, F., and Wang, Y. 2011. Genome-wide identification of Phytophthora sojae SNARE genes and functional characterization of the conserved SNARE PsYKT6. Fungal Genet. Biol. 48:241-251.

Zhou, L., Zhao, J., Guo, W., and Zhang, T. 2013. Functional analysis of autophagy genes via Agrobacterium-mediated transformation in the vascular Wilt fungus Verticillium dahliae. J. Genet. Genomics 40:421-431.

Zhou, T. T., Zhao, Y. L., and Guo, H. S. 2017. Secretory proteins are delivered to the septin-organized penetration interface during root infection by Verticillium dahliae. PLoS Pathog. 13:e1006275.

Zieske, L. R. 2006. A perspective on the use of iTRAQ reagent technology for protein complex and profiling studies. J. Exp. Bot. 57:1501-1508.

\section{AUTHOR-RECOMMENDED INTERNET RESOURCES}

Gene Ontology (GO) Consortium database: http://www.geneontology.org PHI-base database: http://www.phi-base.org 